\title{
Broadcast Scheduling in Battery-Free Wireless Sensor Networks
}

\author{
TONGXIN ZHU, JIANZHONG LI, and HONG GAO, Harbin Institute of Technology, China \\ YINGSHU LI, Georgia State University, USA
}

\begin{abstract}
Battery-Free Wireless Sensor Networks (BF-WSNs) are newly emerging Wireless Sensor Networks (WSNs) to break through the energy limitations of traditional WSNs. In BF-WSNs, the broadcast scheduling problem is more challenging than that in traditional WSNs. This article investigates the broadcast scheduling problem in BF-WSNs with the purpose of minimizing broadcast latency. The Minimum-Latency Broadcast Scheduling problem in BF-WSNs (MLBS-BF) is formally defined and its NP-hardness is proved. Three approximation algorithms for solving the MLBS-BF problem are proposed. The broadcast latency of the broadcast schedules produced by the proposed algorithms is analyzed. The correctness and approximation ratio of the proposed algorithms are also proved. Finally, extensive simulations are conducted to evaluate the performances of the proposed algorithms. The simulation results show that the proposed algorithms have high performance.
\end{abstract}

CCS Concepts: • Networks $\rightarrow$ Sensor networks;

Additional Key Words and Phrases: Battery-free wireless sensor networks, broadcast scheduling, minimum broadcast latency

\section{ACM Reference format:}

Tongxin Zhu, Jianzhong Li, Hong Gao, and Yingshu Li. 2019. Broadcast Scheduling in Battery-Free Wireless Sensor Networks. ACM Trans. Sen. Netw. 15, 4, Article 49 (September 2019), 34 pages.

https://doi.org/10.1145/3356472

\section{INTRODUCTION}

Wireless Sensor Networks (WSNs) are widely employed in variety of applications, such as military surveillance [1, 19], environmental monitoring [11, 23, 30], traffic monitoring [2, 33], and structural health monitoring $[28,50]$, and so on. Sensors in traditional WSNs are powered by batteries. To maintain long network lifetime, batteries usually need to be replaced. However, it is infeasible to replace batteries in many applications where sensors may not be accessible by human beings. Besides, the leakage of batteries may lead to environmental problems. To address such a limitation of traditional WSNs, Battery-Free Wireless Sensor Networks (BF-WSNs) emerge [38, 40]. In

This work is partly supported by the National Natural Science Foundation of China under Grant No. 61632010 and 61832003 , the National Science Foundation (NSF) under Grant No. 1741277 and No. 1829674.

Authors' addresses: T. Zhu, J. Li (corresponding author), and H. Gao, Harbin Institute of Technology, School of Computer Science and Technology, Harbin, Heilongjiang, 150001, China; emails: zhutongxin@stu.hit.edu.cn, \{lijzh, honggao\}@ hit.edu.cn; Y. Li, Georgia State University, Department of Computer Science, Atlanta, GA, 30303, USA; email: yili@gsu.edu. Permission to make digital or hard copies of all or part of this work for personal or classroom use is granted without fee provided that copies are not made or distributed for profit or commercial advantage and that copies bear this notice and the full citation on the first page. Copyrights for components of this work owned by others than ACM must be honored. Abstracting with credit is permitted. To copy otherwise, or republish, to post on servers or to redistribute to lists, requires prior specific permission and/or a fee. Request permissions from permissions@acm.org.

(C) 2019 Association for Computing Machinery.

1550-4859/2019/09-ART49 \$15.00

https://doi.org/10.1145/3356472 
BF-WSNs, sensors do not need batteries but survive through harvesting energy from other energy sources in their ambient environment including solar power, wind power, radio frequency (RF) signals, and so on $[9,37,39]$. Instead, each sensor is equipped with a capacitor that can be unlimitedly recharged for energy storage. From the energy aspect, BF-WSNs have infinite network lifetime. Because of the energy characteristics of the BF-WSNs, existing data processing algorithms $[7,12$, $13,20,52]$ for battery-powered WSNs are not applicable to BF-WSNs any more.

Broadcast is an essential operation in WSNs, which is to disseminate source data from a source sensor node to all the other sensor nodes in a WSN. Each sensor node in a WSN has a transmission range, where sensor nodes within its transmission range can receive the data sent by it. However, the transmission range is limited and is usually much less than the monitoring range of a WSN. Then, there are many intermediate sensor nodes helping to relay the source data in a broadcast operation. Besides, some sensor nodes cannot transmit data simultaneously due to signal collisions. Therefore, it is necessary to schedule the transmissions of all intermediate sensor nodes in a broadcast operation. One of the most popular problem in broadcast scheduling is the minimum-latency broadcast scheduling (MLBS) problem, whose purpose is to minimize the broadcast latency while ensuring all transmissions in the broadcast operation is collision-free.

The MLBS problem is NP-hard [18]. Many approximation algorithms have been proposed accordingly, which can be divided into two categories. The algorithms in the first category solve the MLBS problem in traditional WSNs [10, 17, 18, 24, 25, 32, 43], with the assumption that all sensor nodes are always active in their lifetime. The algorithms in the second category solve the MLBS problem in duty-cycled WSNs [21, 22, 26, 29, 41, 46, 47], with the assumption that every sensor node can be assigned a fixed number of time slots during its lifetime for receiving data and can be woken up at any time to transmit data. A duty-cycled WSN is a special WSN where every sensor node switches between the active state and the sleep state to save energy. Unfortunately, all the aforementioned algorithms are not suitable for BF-WSNs as explained below.

First, the algorithms in the first category assume that all sensor nodes are always active in their lifetime. This assumption is reasonable in battery-powered WSNs but is not realistic in BF-WSNs. Since sensor nodes in BF-WSNs harvest energy from energy sources in their ambient environment, the energy harvesting rate of each sensor node depends on multiple factors (e.g., the distance from the energy source, the energy output power of the energy source, etc.) and is finite. Besides, the energy capacity of each battery-free sensor node is limited by its equipped capacitor. Therefore, the energy stored in battery-free sensor nodes may not always meet the energy requirements of transmitting or receiving data. That means battery-free sensor nodes are not always active in BF-WSNs.

Second, the assumption of the algorithms in the second category that sensor nodes can be assigned fixed time slots to receive data and can be woken up at any time to transmit data is also not reasonable in BF-WSNs. Whether a battery-free sensor node can transmit or receive data depends on its stored energy. And the energy stored in a battery-free sensor node relies on its energy harvesting rate, its energy capacity, and its energy consumption rate. Therefore, battery-free sensor nodes cannot be assigned fixed time slots to receive data without considering their energy status. Besides, a battery-free sensor node cannot be waken up at any time to transmit data, since the energy stored in it cannot always meet the energy requirements of transmitting data.

All such facts indicate that the MLBS problem in BF-WSNs is more challenging than the MLBS problem in traditional WSNs and duty-cycled WSNs. Battery-free sensor nodes harvest energy from energy sources in their ambient environment that are not always stable. Moreover, the energy harvesting rate of a battery-free sensor node is uncontrollable and is usually smaller than its energy consumption rate. Then the biggest challenge of the MLBS problem in BF-WSNs is that battery-free sensor nodes are often out of service in BF-WSNs and any feasible broadcast 
schedule for BF-WSNs cannot ignore the energy status of battery-free sensor nodes. In this article, we investigates the MLBS problem in BF-WSNs. To address the above challenge, we apply some energy prediction methods to estimate the energy status of every battery-free sensor node and estimate the active times of battery-free sensor nodes. Then, the energy status and active times of every batter-free sensor node are taken into consideration in deriving broadcast schedules. To the best of our knowledge, this is the first work to study the MLBS problem in BF-WSNs. The main contributions of the article are as follows.

1. The Minimum-Latency Broadcast Scheduling problem in Battery-Free Wireless Sensor Networks (MLBS-BF) is first proposed and formally defined. The NP-hardness of the MLBS$\mathrm{BF}$ problem is proved.

2. Three approximation algorithms for MLBS-BF problem under protocol interference model with latency at most $16 R-15+T_{\max }$ are proposed, where $R$ is the depth of the breadthfirst searching tree, rooted at the source node, of the input BF-WSN, and $T_{\max }=$ $\max \left\{T_{v}\left(E_{f}\right) \mid v \in V\right\}$, where $T_{v}\left(E_{f}\right)$ is the time consumption for battery-free sensor node $v$ to harvest $E_{f}$ amount of energy required for $v$ to receive and relay the broadcast data packet.

3. The latency of the broadcast schedules produced by the above three algorithms is analyzed, and the correctness and approximation ratio of these algorithms are proved theoretically.

4. The MLBS-BF problem under physical interference model is investigated and the algorithm for generating broadcast schedules for BF-WSNs under physical interference model is proposed.

5. Extensive simulations are carried out to evaluate the performances of the proposed algorithms. The simulation results show that the proposed algorithms have high performances.

The organization of the article is as follows. Section 2 defines the MLBS-BF problem in BF-WSNs and proves the NP-hardness of the problem. Section 3 proposes three approximation algorithms for solving the MLBS-BF problem in BF-WSNs under protocol interference model. Section 4 analyzes the broadcast latency of the broadcast schedules produced by the proposed algorithms and proves the approximation ratio of the proposed algorithms. Section 5 discusses the broadcast scheduling for BF-WSNs under physical interference model. Section 6 shows the simulation results and analyzes the performances of the proposed algorithms. Section 7 reviews the related works. Section 8 concludes the article.

\section{PROBLEM DEFINITION}

\subsection{Network Model}

A BF-WSN has a set of $n$ sensor nodes that are randomly deployed in a region, denoted as $V=\left\{v_{1}, v_{2}, \ldots, v_{n}\right\}$. The source sensor node of a broadcast is denoted as $s$, where $s \in V$. Every sensor node in a BF-WSN is battery-free and is powered by the energy sources in its ambient environment. Different from traditional WSNs, a BF-WSN has infinite lifetime in terms of energy. We apply the fixed data-rate channel model, where each battery-free sensor node has a fixed transmission power $P$. Thus, each battery-free sensor node can transmit its data packets over a common wireless channel with bandwidth $W$ bits/second and all battery-free sensor nodes have the same transmission range denoted by $r$. We assume that the source sensor node $s$ has a broadcast data packet of $B$ bits. Then the time consumption for receiving and transmitting a data packet of $B$ bits are denoted as $\Delta_{r}$ and $\Delta_{t}$, where $\Delta_{r}=\Delta_{t}=B / W$. In this article, we use $\Delta=B / W$ to denote the measurement unit of time. 
The network topology of a BF-WSN can be expressed by a graph $G(V, E)$, where $V=$ $\left\{v_{1}, v_{2}, \ldots, v_{n}\right\}$ is the set of battery-free sensor nodes, and $\left(v_{i}, v_{j}\right) \in E$ if and only if $\operatorname{dist}\left(v_{i}, v_{j}\right) \leq r$, where $\operatorname{dist}\left(v_{i}, v_{j}\right)$ is the Euclidean distance between $v_{i}$ and $v_{j}$. The sensor nodes within the transmission range of sensor node $v_{i}$ are called the neighbors of $v_{i}$. The set of all the neighbors of $v_{i}$ is denoted by $N\left(v_{i}\right)$, where $N\left(v_{i}\right)=\left\{v_{j} \mid v_{j} \in V,\left(v_{j}, v_{i}\right) \in E\right\}$.

In this article, two interference models are considered, the protocol interference model and the physical interference model.

(1) Under the protocol interference model, all sensor nodes have a uniform interference range. For simplicity, the interference range is the same as the the transmission range $r$ in this article. First, each sensor node cannot transmit and receive data simultaneously according to its half-duplex mode. Second, some sensor nodes cannot transmit simultaneously on account of collisions. When sensor node $v_{i}$ transmits its data to sensor node $v_{j}$, the transmission is collision-free when $v_{j}$ is in the transmission range of its intended transmitter $v_{i}$ and is not in the interference ranges of other non-intended simultaneous transmitters. That is, $\operatorname{dist}\left(v_{i}, v_{j}\right) \leq r$ but $\forall v_{k} \in I$, $\operatorname{dist}\left(v_{k}, v_{j}\right)>r$, where $\operatorname{dist}\left(v_{i}, v_{j}\right)$ is the Euclidean distance between $v_{i}$ and $v_{j}$ and $I$ is the set of sensor nodes (except $v_{i}$ ) transmitting simultaneously with $v_{i}$.

(2) Under the physical interference model, a sensor node can successfully receive a data packet only if the ratio between the received signal strength and the noise plus the interference caused by all simultaneously transmitting sensor nodes, the Signal-to-Interferenceplus-Noise Ratio (SINR) value, is greater than a threshold. That is, a sensor node $v_{j}$ can successfully receive the data packet transmitted by $v_{i}$ if the SINR value between them is greater than a threshold, i.e.,

$$
\operatorname{SINR}_{i j}=\frac{P \cdot \operatorname{dist}\left(v_{i}, v_{j}\right)^{-\alpha}}{N_{0}+\sum_{v_{k} \in I} P \cdot \operatorname{dist}\left(v_{k}, v_{j}\right)^{-\alpha}} \geq \beta,
$$

where $\operatorname{dist}\left(v_{i}, v_{j}\right)$ is the Euclidean distance between $v_{i}$ and $v_{j}, \alpha$ is the path loss exponent and usually $2 \leq \alpha \leq 6, N_{0}$ is the background noise, $I$ is the set of sensor nodes (except $v_{i}$ ) transmitting simultaneously with $v_{i}$, and $\beta$ is a hardware determined threshold value that is no less than 1 .

\subsection{Energy Model}

Each battery-free sensor node in a BF-WSN harvests energy from the energy sources in its ambient environment, such as solar, wind, RF signal, and so on. The time consumption for a battery-free sensor node to harvest a certain amount of energy depends on many factors, such as the distance between the battery-free sensor node and the energy source in its ambient environment, the stored energy in the battery-free sensor node and the energy output power of different energy sources, and so on. We use $T_{v}(e)$ to denote the time consumption function for battery-free sensor node $v$ to harvest $e$ amount of energy from 0 . Usually, $T_{v}(e)$ is a non-decreasing and convex function [5].

$T_{v}(e)$ is an important parameter in broadcast scheduling for BF-WSNs, since it indicates the time at which a battery-free sensor node has enough energy to transmit or receive data. Fortunately, there exists a large number of works focusing on building energy prediction models for sensor nodes harvesting energy from different energy sources. For solar energy harvesting sensor networks, the Exponentially Weighted Moving-Average is a widely used energy prediction model [27]. Another energy prediction model, the Weather-Conditioned Moving Average [36], is proposed for solar energy harvesting sensor networks in the case of frequently changing weather 


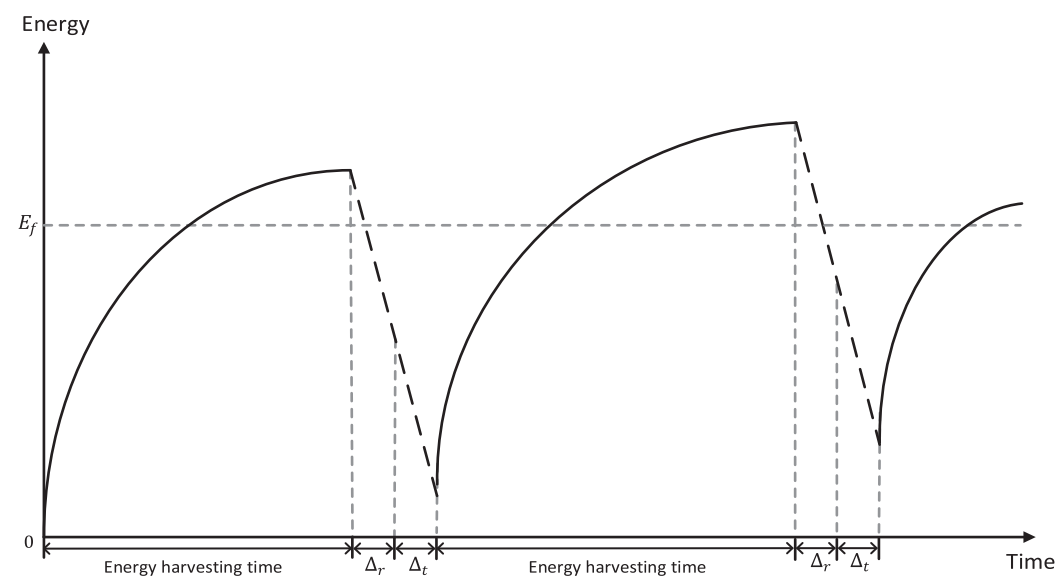

Fig. 1. The stored energy in $v$ varying with time.

conditions. Different solar energy prediction models are compared in Reference [3], whose simulation results show that the minimum average error of the models is less than $10 \%$. A variety of energy prediction models for wind energy harvesting sensor networks are reviewed and compared in Reference [16], and it is indicated that some wind energy prediction models can produce quite low errors. Besides, a novel energy prediction model, named Pro-Energy, is proposed in Reference [8] for both solar and wind energy harvesting. Compared with other energy prediction models, Pro-energy improves the prediction accuracy for up to $60 \%$. For RF energy harvesting sensor networks in the case of the dedicated RF transmitters being applied to generate stable RF signals, the Friis transmission equation can be used as the energy prediction model [31, 34, 35]. For RF energy harvesting sensor networks in the case of the RF energy transmitters being uncontrolled or unstable such as radio and television broadcasting, a genetic machine learning approach is proposed to predict harvested energy [14].

According to the energy prediction models for BF-WSNs with different energy sources mentioned above, the time consumption function $T_{v}(e)$ for a battery-free sensor node $v$ can be estimated with high accuracy. Therefore, it is reasonable to assume that $T_{v}(e)$ for a battery-free sensor node in the broadcast scheduling period can be estimated with high accuracy. In rest of this article, we assume that $T_{v}(e)$ is known in advance in a broadcast scheduling period.

A battery-free sensor node periodically switches among three states, charging state, receiving state and transmitting state. A battery-free sensor node harvests energy from the ambient environment in the charging state, and receives or transmits data only in the receiving state or transmitting state. It is well known that radio communication consumes the vast majority of energy while sensing consumes negligible energy $[15,45]$. Thus, we ignore the energy consumption for sensing in this article.

Generally, the energy in a battery-free sensor node $v$ varies with time, which can be described by a curve as shown in Figure 1. The solid lines present the process of energy harvesting while the dotted lines present the energy consumption of data reception and transmission. The energy consumption for a battery-free sensor node to receive and relay a broadcast data packet of $B$ bits is denoted by $E_{f}$. The time consumption for a battery-free sensor node to harvest $E_{f}$ amount of energy from 0 is $T_{v}\left(E_{f}\right)$, where the measurement unit of $T_{v}\left(E_{f}\right)$ is $\Delta=B / W$. We use $T_{\max }$ to denote the maximum time consumption for all battery-free sensor nodes to harvest $E_{f}$ amount of energy, i.e., $T_{\max }=\max \left\{T_{v}\left(E_{f}\right) \mid v \in V\right\}$.

The symbols used in this section are summarized in Table 1. 
Table 1. Symbol Table

\begin{tabular}{ll}
\hline Symbol & \multicolumn{1}{c}{ Description } \\
\hline$V=\left\{v_{1}, v_{2}, \ldots, v_{n}\right\}$ & The set of battery-free sensor nodes. \\
$r$ & The transmission power of battery-free sensor nodes. \\
$r$ & The transmission range and interference range of battery \\
& -free sensor nodes. \\
& The Euclidean distance between sensor nodes $v_{i}$ and $v_{j}$. \\
dist $\left(v_{i}, v_{j}\right)$ & The network topology of a BF-WSN. \\
$G(V, E)$ & The set of neighbors of sensor node $v_{i}$. \\
$N\left(v_{i}\right)$ & The size of a broadcast data packet. \\
\hline$B$ & The bandwidth of the wireless channel. \\
$W$ & The time consumption for receiving the broadcast data \\
$\Delta_{r}$ & packet of $B$ bits, where $\Delta_{r}=B / W$. \\
& The time consumption for transmitting the broadcast \\
$\Delta_{t}$ & data packet of $B$ bits, where $\Delta_{t}=B / W$. \\
& The measurement unit of time, where $\Delta=\Delta_{r}=\Delta_{t}$. \\
$\Delta$ & The energy consumption for a sensor node to receive \\
$E_{f}$ & and relay a data packet of $B$ bits. \\
& The time consumption for battery-free sensor node $v$ to \\
$T_{v}(e)$ & harvest $e$ amount of energy from 0. \\
& The maximum time consumption for all battery-free \\
$T_{m a x}=\max \left\{T_{v}\left(E_{f}\right) \mid v \in V\right\}$ & sensor nodes to harvest $E_{f}$ amount of energy. \\
\hline$s$ & The source sensor node of a broadcast, and $s \in V$. \\
$t_{0}$ & The initial time of a broadcast. \\
$S C H=\left\{\left\langle\{s\}, t_{1}\right\rangle, \ldots,\left\langle s_{l}, t_{l}\right\rangle\right\}$ & A broadcast schedule of the BF-WSN. \\
$L_{a}$ & The broadcast latency of broadcast schedule $S C H=$ \\
& $\left\{\left\langle\{s\}, t_{1}\right\rangle, \ldots,\left\langle s, t_{l}\right\rangle\right\}$, where $L_{a}=t_{l}+\Delta-t_{0}$.
\end{tabular}

\subsection{Problem Statement}

This article focuses on the problem of single-source broadcast in BF-WSNs, which is to deliver a broadcast data packet of the source sensor node $s$ to all the other battery-free sensor nodes in a BF-WSN. A single-source collision-free broadcast schedule is defined as follows.

Definition 1 (Single-Source Collision-Free Broadcast Schedule in BF-WSNs). A single-source collision-free broadcast schedule is a sequence of binary tuples $S C H=\left\{\left\langle s_{1}, t_{1}\right\rangle,\left\langle s_{2}, t_{2}\right\rangle, \ldots,\left\langle s_{l}, t_{l}\right\rangle\right\}$, where $\left(s_{i}, t_{i}\right)(1 \leq i \leq l)$ represents that battery-free sensor nodes in $s_{i} \subset V$ transmit the broadcast data packet in time interval $\left[t_{i}, t_{i}+\Delta\right]$. Any single-source collision-free broadcast schedule satisfies the following conditions.

(1) $s_{1}=\{s\}$;

(2) $\bigcup_{i=1}^{l} s_{i} \subset V$;

(3) Each battery-free sensor node only transmits once in a broadcast, i.e., $s_{i} \cap s_{j}=\emptyset$, where $1 \leq i \leq l, 1 \leq j \leq l$ and $i \neq j$;

(4) The initial time of a broadcast is $t_{0}$ and $t_{0} \leq t_{1}<\cdots<t_{l}$;

(5) All the battery-free sensor nodes in $s_{i}$ can transmit the broadcast data packet simultaneously without collisions under protocol interference model or physical interference model;

(6) All the battery-free sensor nodes in a BF-WSN have received the broadcast data packet at time $t_{l}+\Delta$, where the broadcast latency is $L a=t_{l}+\Delta-t_{0}$. 
The Minimum-Latency Broadcast Scheduling problem in BF-WSNs (MLBS-BF) is to compute a single-source collision-free broadcast schedule for a BF-WSN with minimum broadcast latency, which can be formulated as follows.

\section{Input:}

1. $G(V, E)$, the topology graph of a BF-WSN,

2. $s$, the source sensor node of a broadcast,

3. $t_{0}$, the initial time of the broadcast,

4. $T_{v_{i}}(e)(1 \leq i \leq n)$, the time consumption functions of battery-free sensor nodes, and

5. $E_{f}$, the energy consumption for each battery-free sensor node to receive and relay the broadcast data packet.

\section{Output:}

$S C H=\left\{\left\langle\{s\}, t_{1}\right\rangle,\left\langle s_{2}, t_{2}\right\rangle, \ldots,\left\langle s_{l}, t_{l}\right\rangle\right\}$, the single-source collision-free broadcast schedule of the BF-WSN described by $G(V, E)$ such that the broadcast latency $L a=t_{l}+\Delta-t_{0}$ is minimized.

Theorem 1. The Minimum-Latency Broadcast Scheduling problem in BF-WSN (MLBS-BF) is NPhard.

Proof. The following problem, denoted by Sub-MLBS-BF, is a special case of the MLBS-BF problem.

\section{Input:}

1. $t_{0}$, the initial time of the broadcast,

2. $G(V, E)$, the topology graph of a BF-WSN, in which every sensor node has $E$ amount of initial energy in its capacitor at time $t_{0}$,

3. $s$, the source sensor node of a broadcast,

4. $T_{v_{i}}(e)=+\infty(1 \leq i \leq n)$, the time consumption functions of all sensor nodes, which means the sensor nodes in the BF-WSN cannot be charged, and

5. $E_{f}$, the energy consumption for each sensor node to receive and relay the broadcast data packet, where $E_{f} \leq E$.

\section{Output:}

$S C H=\left\{\left\langle\{s\}, t_{1}\right\rangle,\left\langle s_{2}, t_{2}\right\rangle, \ldots,\left\langle s_{l}, t_{l}\right\rangle\right\}$, the single-source collision-free broadcast schedule of the BF-WSN described by $G(V, E)$ such that the broadcast latency $L a=t_{l}+\Delta-t_{0}$ is minimized.

It is obvious that the Sub-MLBS-BF problem is equivalent to the MLBS problem in traditional WSNs. It has been proved that the MLBS problem in traditional WSNs under the protocol interference model is NP-hard [18]. And the physical interference model can be regarded as a more general case and is therefore also NP-hard [24]. Thus, the MLBS-BF problem is NP-hard.

\section{ALGORITHMS FOR SOLVING THE MLBS-BF PROBLEM}

In this section, we propose three approximation algorithms for solving the MLBS-BF problem, named as Sequence Broadcast Scheduling (SBS) algorithm, Waiting Time Shortened Broadcast Scheduling (WTSBS) algorithm, and Enhanced Waiting Time Shortened Broadcast Scheduling (EWTSBS) algorithm, respectively.

\subsection{The Sequence Broadcast Scheduling Algorithm}

The SBS algorithm is an approximation algorithm for deriving a collision-free broadcast schedule $S C H=\left\{\left\langle s_{1}, t_{1}\right\rangle,\left\langle s_{2}, t_{2}\right\rangle, \ldots,\left\langle s_{l}, t_{l}\right\rangle\right\}$ to reduce broadcast latency as much as possible. The SBS algorithm consists of two phases. The first phase is to generate layered transmission sequences from $G(V, E)$ of the input BF-WSN, which builds a communication frame for passing the broadcast data 


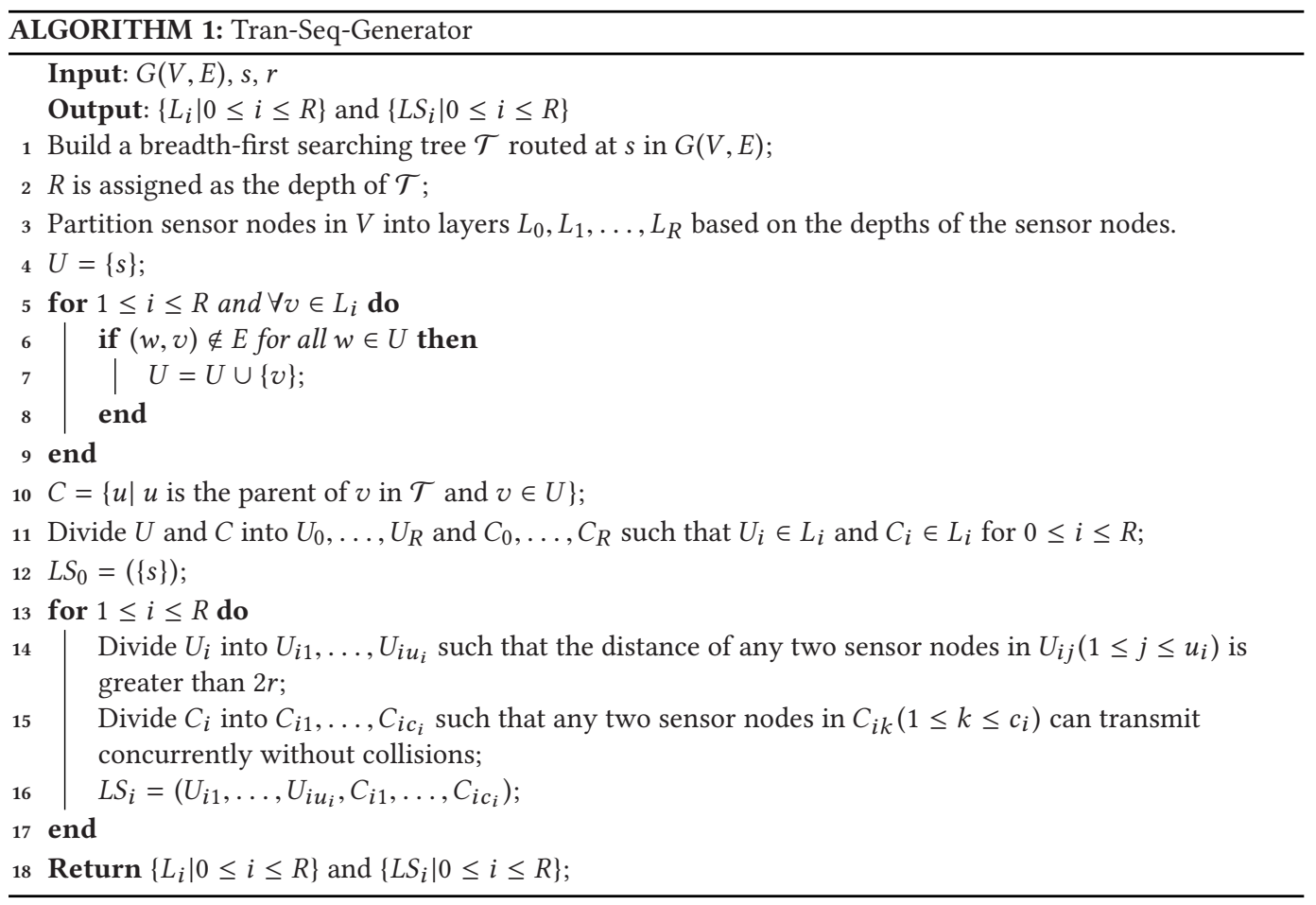

packet initiated by the source sensor node $s$. The second phase is to assign transmission times for sensor nodes in each transmission sequence generated in the first phase. The details of these two phases are as follows.

3.1.1 Phase One: Transmission Sequence Generation. In the first phase, we divide the BF-WSNs into layers and generate the transmission sequence of battery-free sensor nodes layer by layer. The transmission sequence is generated without considering energy of battery-free sensor nodes and just builds a communication frame for passing the broadcast data packet initiated by the source sensor node. Therefore, the first phase can be implemented by the idea in Reference [25], which studies the problem of minimum-latency broadcast scheduling in battery-powered WSNs. The work in Reference [25] divides the WSNs into layers by breadth-first searching and generates collision-free transmission sequence of broadcast by connected dominating set. We also apply the technique of breadth-first searching and connected dominating set to generate transmission sequence. The following is the outline of the algorithm, named as Tran-Seq-Generator, shown in Algorithm 1. Tran-Seq-Generator is composed by the following three steps.

Step 1 (Lines 1-3). Partition all battery-free sensor nodes into layers. First, build a tree $\mathcal{T}$ rooted at $s$ by breadth-first searching on the topology graph $G(V, E)$ of the input BF-WSN. The depth of a sensor node $v$ in $\mathcal{T}$ is the number of hops between $s$ and $v$. Then, the sensor nodes in $V$ are partitioned into layers $L_{0}, L_{1}, \ldots, L_{R}$, where $R$ is the depth of $\mathcal{T}$ and $L_{i}$ contains all sensor nodes with depth $i$ in $\mathcal{T}$. Obviously, only source sensor node $s$ is in layer $L_{0}$.

Step 2 (Lines 4-11). First, obtain the maximal independent set $U$ of $G(V, E)$ by selecting sensor nodes from $\mathcal{T}$ layer by layer as follows. The root $s$ in layer $L_{0}$ is first put into $U$. Then, for $1 \leq i \leq R$ and $v \in L_{i}, v$ is put into $U$ if and only if $\forall w \in U,(w, v) \notin E$. $U$ can be divided into $U_{0}, U_{1}, \ldots, U_{R}$ 


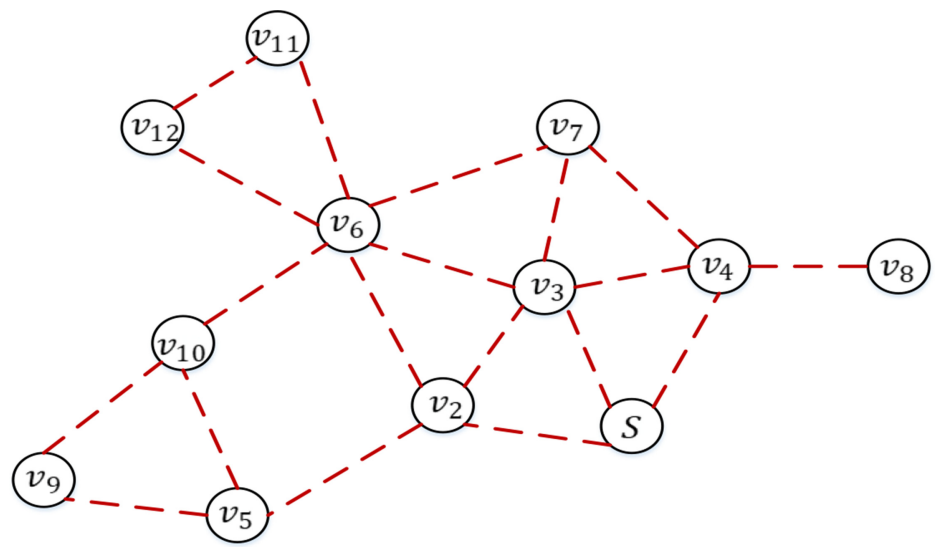

Fig. 2. The topology graph $G(V, E)$ of a BF-WSN with 12 battery-free sensor nodes.

such that $U_{i} \subseteq L_{i}$ for $0 \leq i \leq R$. Then, compute $C=\{u \mid \exists v \in U$ and $u$ is the parent of $v$ in $\mathcal{T}\}$. Finally, $C$ is also divided into $C_{0}, C_{1}, \ldots, C_{R}$ such that $C_{i} \subseteq L_{i}$ for $0 \leq i \leq R$.

Step 3 (Lines 12-17). Generate the transmission sequence $L S_{i}$ for $0 \leq i \leq R$ as follows. At layer $L_{0}=\{s\}, L S_{0}=(\{s\})$. For $1 \leq i \leq R$, compute $L S_{i}$ at layer $L_{i}$. First, $U_{i}$ is divided into subsets $U_{i 1}, \ldots, U_{i u_{i}}$ such that the distance of any pair of sensor nodes in $U_{i j}\left(1 \leq j \leq u_{i}\right)$ is greater than $2 r$, that is, all sensor nodes in $U_{i j}$ can transmit concurrently without collisions. Second, $C_{i}$ is divided into subsets $C_{i 1}, \ldots, C_{i c_{i}}$ such that sensor nodes in $C_{i k}$ can transmit concurrently without collisions for $1 \leq k \leq c_{i}$. Finally, $L S_{i}=\left(U_{i 1}, \ldots, U_{i u_{i}}, C_{i 1}, \ldots, C_{i c_{i}}\right)$ is generated.

Finally, the transmission sequence $\left\{L S_{i} \mid 0 \leq i \leq R\right\}$ is generated by the first phase. The Tran-SeqGenerator algorithm only considers the network topology of the BF-WSNs instead of energy of battery-free sensors in BF-WSNs. The sink node only collects the network topology to generate the transmission sequence for broadcast scheduling. Since the network topology can be collected by the sink node before broadcast scheduling or even at the initial time of the deployment of the BFWSN. Therefore, once the transmission sequence is generated by the Tran-Seq-Generator, it will not change until the network topology changes. That means, the generated transmission sequence can be applied by multiple broadcast schedules. An example of the process of the first phase is illustrated in Figure 2 and Figure 3. The topology graph of a BF-WSN with 12 battery-free sensor nodes is presented in Figure 2, where the dashed lines represent the edges in $G(V, E)$. The breadthfirst searching tree of the BF-WSN is presented in Figure 3. The blue nodes in Figure 3 represent the sensor nodes in the maximal independent set and the orange nodes in Figure 3 represent the sensor nodes in $C$, i.e., $U=\left\{s, v_{5}, v_{6}, v_{8}\right\}$ and $C=\left\{v_{2}, v_{4}\right\}$. Besides, $U$ is divided into $U_{0}=\{s\}$ and $U_{2}=$ $\left\{v_{5}, v_{6}, v_{8}\right\}$ and $C$ is divided into $C_{1}=\left\{v_{2}, v_{4}\right\}$ according to the layers. Therefore, the transmission sequence is $L S_{0}=(\{s\}), L S_{1}=\left(C_{11}, C_{12}\right)=\left(\left\{v_{2}\right\},\left\{v_{4}\right\}\right), L S_{2}=\left(U_{21}, U_{22}\right)=\left(\left\{v_{5}, v_{8}\right\},\left\{v_{6}\right\}\right)$.

3.1.2 Phase Two: Transmission Time Assignment. The output $\left\{L S_{i} \mid 0 \leq i \leq R\right\}$ of the first phase is the basis of the second phase of the SBS algorithm. The second phase aims at assigning transmission times for sensor nodes in every element of $L S_{i}$ for each $0 \leq i \leq R$. The features of set $\left\{L S_{i} \mid 0 \leq i \leq R\right\}$ suggest the following hints for assigning transmission times for sensor nodes.

1. Only sensor nodes in $U$ and $C$ should be scheduled to relay the broadcast data packet and other sensor nodes just receive the broadcast data packet from them.

2. Sensor nodes in $U_{i-1} \cup C_{i-1}$ should be scheduled earlier than the ones in $U_{i} \cup C_{i}$ for all $1 \leq i \leq R$.

3. Sensor nodes in $U_{i}$ should be scheduled earlier than the ones in $C_{i}$ for $0 \leq i \leq R$. 


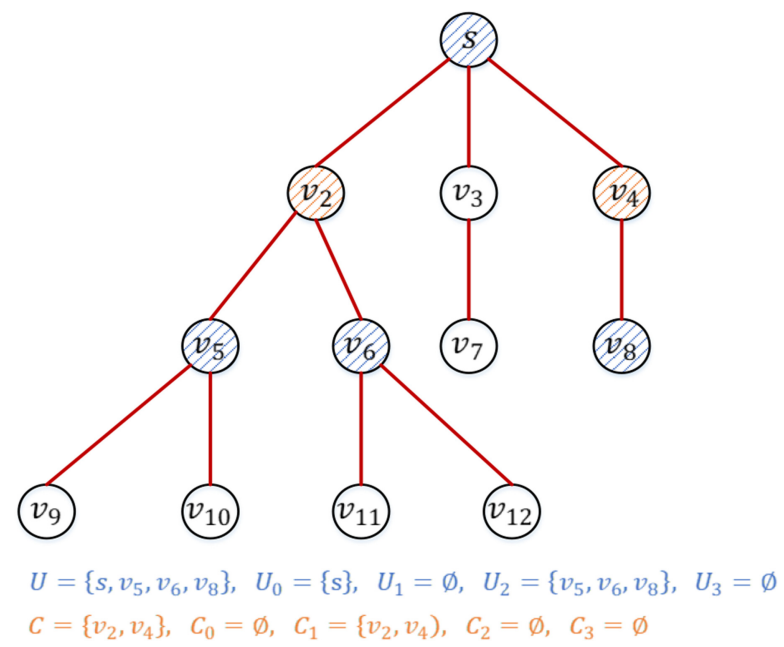

Fig. 3. The breadth-first searching tree $\mathcal{T}$ of graph $G(V, E)$.

Based on the hints, the algorithm of the second phase, named as Tran-Time-Assigner1, shown in Algorithm 2, is to assign transmission times for sensor nodes in every element of $L S_{i}$ for each $0 \leq i \leq R$ and then generate a broadcast schedule $S C H_{S B S}$. The second phase is difficult, because the energy status of each battery-free sensor node must be taken into account.

The broadcast is initiated by the source sensor node $s$ at time $t_{0}$, and $S C H_{S B S}$ is initialized as $S C H_{S B S}=\emptyset$. At time $t_{0}, s$ has enough energy to work. For $0 \leq i \leq R$, Tran-Time-Assigner1 assigns transmission times for sensor nodes in each element of the transmission sequence $L S_{i}=$ $\left(U_{i 1}, \ldots, U_{i u_{i}}, C_{i 1}, \ldots, C_{i c_{i}}\right)$ by the following three steps.

Step 1 (Lines 2-4, 8-13, and 19-24). Build the receiving set $R_{v}$, which is the set of sensor nodes that need to receive the broadcast data packet from $v$, for each sensor node $v$ in each $U_{i j}\left(1 \leq j \leq u_{i}\right)$ or $C_{i k}\left(1 \leq k \leq c_{i}\right)$ of $L S_{i}$. The earliest possible transmission time for $v$ depends on $R_{v}$.

Sensor nodes in $R_{v}$ cannot always be awake, since some of them may not have enough energy for working. A sensor node wakes up if it has harvested more than $E_{f}$ energy, which can afford it to receive and relay the broadcast data packet. Due to the different energy harvesting rates, sensor nodes in $R_{v}$ may wake up at different time. There are two strategies for $v$ to transmit the broadcast data packet. The first one is to transmit the broadcast data packet every time a sensor node in $R_{v}$ wakes up. The second one is to transmit the broadcast data packet only one time when all sensor nodes in $R_{v}$ are awake. The first strategy has longer latency than the second one, since the first strategy requires $v$ to transmit the broadcast data packet multiple times and thus need to harvest energy many times. Thus the second strategy is adopted. Initially, $\forall v \in U \cup C, R_{v}=\emptyset$. The receiving set of each sensor node in each $U_{i j}\left(1 \leq j \leq u_{i}\right)$ or $C_{i k}\left(1 \leq k \leq c_{i}\right)$ of $L S_{i}$ is built as follows.

First, for every sensor node $v$ in each element of $L S_{i}, R_{v}=N(v) \cap\left(L_{i} \cup L_{i+1}\right)-\cup_{u \in U \cup C} R_{u}$ that includes all neighbors of $v$ in layer $L_{i}$ and $L_{i+1}$ sensor nodes that have not be assigned to other receiving sets.

Second, each receiving set $R_{v}$ is pruned to reduce broadcast latency of sensor node $v$. Obviously, if the size of $R_{v}$ is smaller, the time consumption of $v$ to wait for its receivers waking up will be less, and thus the broadcast latency will be reduced. A sensor node $u$ can be removed from $R_{v}$ if $u$ can receive the broadcast data packet from another sensor node whose transmission time is latter than $v$. The concrete method is as follows. In case of $v \in U_{i j}\left(1 \leq j \leq u_{i}\right)$, sensor node $u$ is 


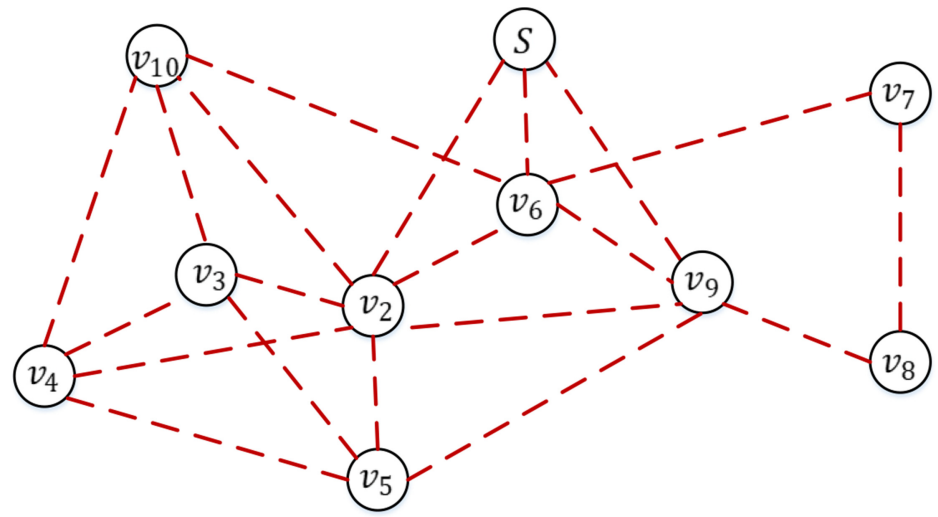

Fig. 4. A BF-WSN with 10 battery-free sensor nodes.

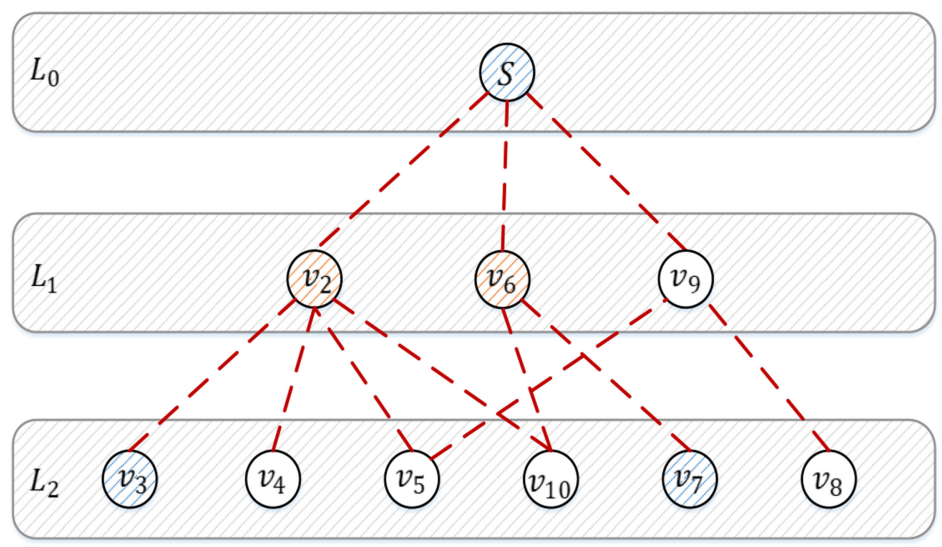

Fig. 5. An example of building the receiving set $R_{v}$.

removed from $R_{v}$ if $u$ satisfies that $u \in L_{i+1}$ and $u \in N\left(\cup_{h=j+1}^{u_{i}} U_{i h} \cup \cup_{g=1}^{c_{i}} C_{i g}\right)$. In case of $v \in C_{i k}$ $\left(1 \leq k \leq c_{i}\right)$, sensor node $u$ is removed from $R_{v}$ if $u$ satisfies that $u \in L_{i+1}$ and $u \in N\left(\bigcup_{g=k+1}^{c_{i}} C_{i g}\right)$.

An example of building the receiving set $R_{v}$ is shown in Figure 4 and Figure 5. A BFWSN with 10 battery-free sensor nodes is shown in Figure 4, where the dashed lines represent the edges between sensor nodes in the topology graph $G(V, E)$. The layered BF-WSN is presented in Figure 5, where only the edges between sensor nodes in different layers are shown for clarity. First, $R_{s}=\left\{v_{2}, v_{6}, v_{9}\right\}$. The transmission sequence of layer $L_{1}$ is $L S_{1}=\left(C_{11}, C_{12}\right)=$ $\left(\left\{v_{2}\right\},\left\{v_{6}\right\}\right)$. Initially, $R_{v_{2}}=N\left(v_{2}\right) \cap\left(L_{1} \cup L_{2}\right)-\cup_{u \in U \cup C} R_{u}=\left\{v_{3}, v_{4}, v_{5}, v_{6}, v_{9}, v_{10}\right\}-\left\{v_{2}, v_{6}, v_{9}\right\}=$ $\left\{v_{3}, v_{4}, v_{5}, v_{10}\right\}$. Since $v_{10} \in L_{2}$ and $v_{10} \in N\left(C_{12}\right)$, which means that $v_{10}$ can receive the data packet from $v_{6} \in C_{12}$ instead of $v_{2}, v_{10}$ is removed from $R_{v_{2}}$. Finally, $R_{v_{2}}$ is $\left\{v_{3}, v_{4}, v_{5}\right\}$. Besides, the receiving set $R_{v_{6}}$ of $v_{6}$ is $\left\{v_{7}, v_{10}\right\}$.

Step 2 (Lines 6-28). Assign transmission times for sensor nodes in each element of $L S_{i}=$ $\left(U_{i 1}, \ldots, U_{i u_{i}}, C_{i 1}, \ldots, C_{i c_{i}}\right)$ based on their receiving sets built in Step 1. In the following, $t^{-}$denotes the latest transmission time that has already been assigned to a sensor node.

First, assign transmission times for sensor nodes in $U_{i j}$ for $1 \leq j \leq u_{i}$ to generate a partial schedule $U T_{i}$. Let $t^{-}$be the latest transmission time that has already been assigned to a sensor node in the elements of $L S_{i-1}$. 


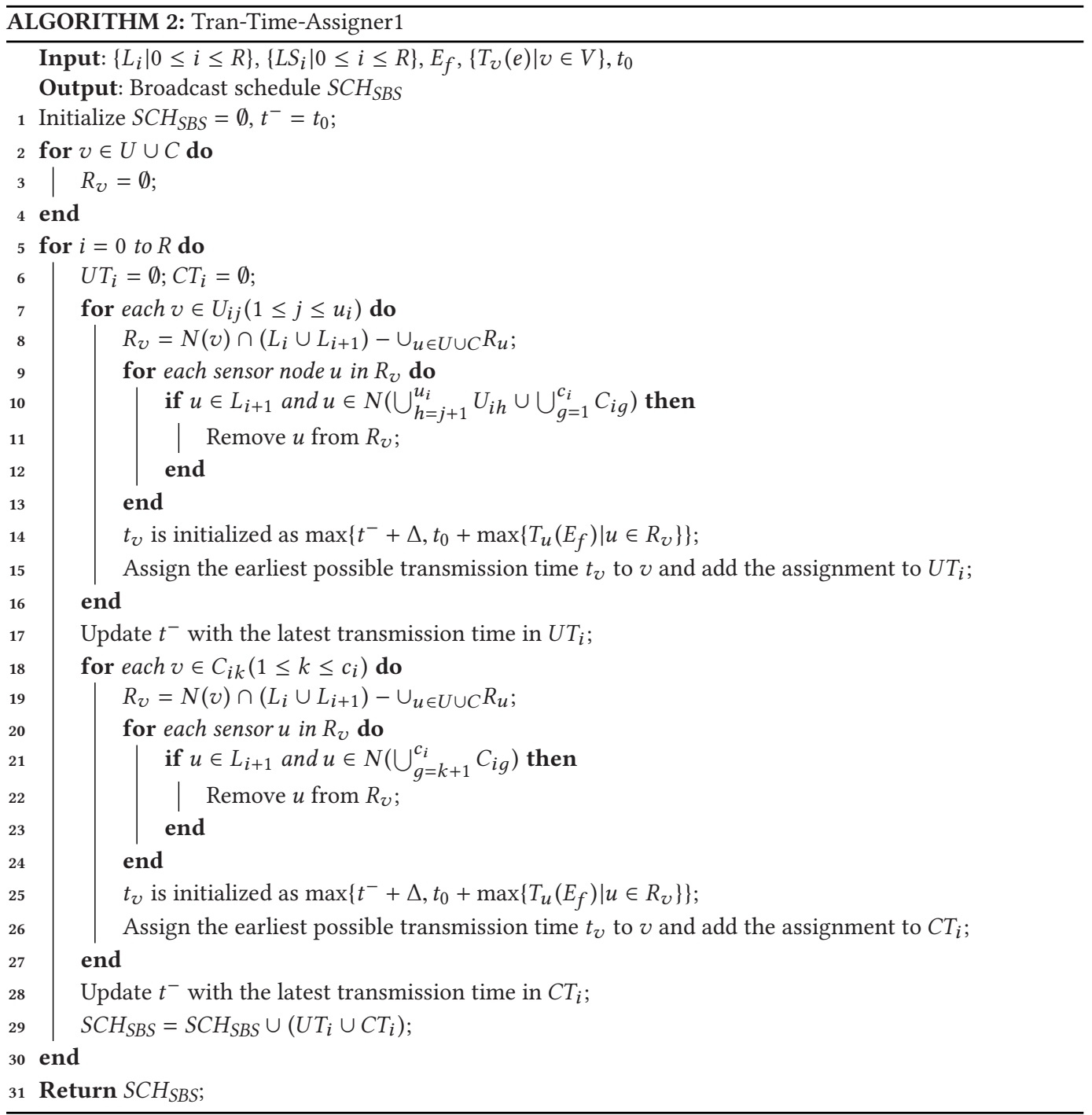

For each sensor node $v \in U_{i j}$, the assigned transmission time of $v$ is denoted as $t_{v}$, which is initialized as $t_{v}=\max \left\{t^{-}+\Delta, t_{0}+\max \left\{T_{u}\left(E_{f}\right) \mid u \in R_{v}\right\}\right\}$. According to the meaning of time $t_{v}$, all sensor nodes in all the elements of $L S_{i-1}$ have completed their transmissions and $v$ must have received the broadcast data packet at time $t^{-}+\Delta$. Besides, $T_{u}\left(E_{f}\right)\left(u \in R_{v}\right)$ denotes the time consumption for $u$ to accumulate enough energy for transmitting and receiving the broadcast data packet. And $T_{u}\left(E_{f}\right)$ is estimated by $u$ through energy prediction methods and is collected at the initial time $t_{0}$. At time $t_{0}+\max \left\{T_{u}\left(E_{f}\right) \mid u \in R_{v}\right\}$, all sensor nodes in $R_{v}$ have harvested $E_{f}$ energy and can be waken up to receive the broadcast data packet from $v$. Therefore, at time $t_{v}, v$ may start to transmit the broadcast data packet. Thus, we can assign a transmission time to $v$ and add the assignment to $U T_{i}$ as follows.

1. If there is not an element $\left\langle S, t_{v}\right\rangle$ in partial schedule $U T_{i}$, which means $v$ can transmit at time $t_{v}$ without collisions, then add $\left\langle\{v\}, t_{v}\right\rangle$ to $U T_{i}$. 


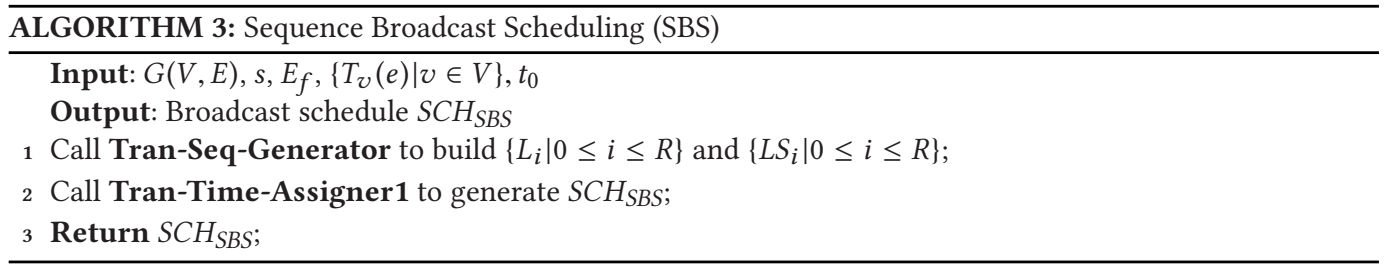

2. If there is an element $\left\langle S, t_{v}\right\rangle \in U T_{i}$ and $S \subseteq U_{i j}$, which means the concurrent transmissions of $v$ and all sensor nodes in $S$ will not cause collisions, then update $\left\langle S, t_{v}\right\rangle$ with $\left\langle S \cup\{v\}, t_{v}\right\rangle$ in $U T_{i}$.

3. If neither of the above two conditions is true, then it means there is a sensor node $w$ transmitting the broadcast data packet at time $t_{v}$ and the concurrent transmissions of $w$ and $v$ will cause collisions. However, $w$ will complete its transmission at time $t_{v}+\Delta$. Thus, increase $t_{v}$ by $\Delta$ and go to 1 .

Second, assign transmission times for sensor nodes in $C_{i k}$ for $1 \leq k \leq c_{i}$ to generate another partial schedule $C T_{i}$. It is similar to the assignment for sensor nodes in $U_{i j}\left(1 \leq j \leq u_{i}\right)$. Let $t^{-}$be the latest transmission time that has already been assigned to a sensor node in the elements of $U T_{i}$.

For each sensor node $v \in C_{i k}$, its transmission time $t_{v}$ is initialized as $t_{v}=\max \left\{t^{-}+\Delta, t_{0}+\right.$ $\left.\max \left\{T_{u}\left(E_{f}\right) \mid u \in R_{v}\right\}\right\}$. Then, assign a transmission time to $v$ and add the assignment into $C T_{i}$ as follows.

1. If there is not an element $\left\langle S, t_{v}\right\rangle$ in partial schedule $C T_{i}$, which means no sensor nodes in $C T_{i}$ will transmit the broadcast data packet at time $t_{v}$ and $v$ can transmit the broadcast data packet at time $t_{v}$ without collisions, then add $\left\langle\{v\}, t_{v}\right\rangle$ to $C T_{i}$.

2. If there is an element $\left\langle S, t_{v}\right\rangle$ in $C T_{i}$ and $S \subseteq C_{i k}$, which means $t_{v}$ can transmit the broadcast data packet at time $t_{v}$ without conflicting with all sensor nodes in $S$, then update $\left\langle S, t_{v}\right\rangle$ with $\left\langle S \cup\{v\}, t_{v}\right\rangle$ in $C T_{i}$.

3. If neither of the above two conditions is true, the next possible transmission time for $v$ is $t_{v}+\Delta$. Then, increase $t_{v}$ by $\Delta$ and go to 1 .

Step 3 (Line 29). Update the broadcast schedule $S C H_{S B S}$ by $S C H_{S B S}=S C H_{S B S} \cup\left(U T_{i} \cup C T_{i}\right)$.

After all sensor nodes in all elements of the transmission sequence $L S_{i}=\left(U_{i 1}, \ldots, U_{i u_{i}}\right.$, $\left.C_{i 1}, \ldots, C_{i c_{i}}\right)$ have been assigned transmission times, the collision-free broadcast schedule for the BF-WSN is generated. The SBS algorithm consists of Tran-Seq-Generator algorithm and TranTime-Assigner1 algorithm as shown in Algorithm 3.

The SBS algorithm proposed in this article can generate single-source collision-free broadcast schedules for BF-WSNs. The reasons why the existing broadcast scheduling algorithms are no longer feasible for BF-WSNs and the challenges of broadcast scheduling in BF-WSNs have been introduced in Section 1. The SBS algorithm addresses these challenges by considering the energy status of every battery-free sensor node in BF-WSNs. Firstly, the set of battery-free sensor nodes that need to relay the broadcast data packet is computed, i.e., $U \cup C$. Then, the receiving set $R_{v}$ of each battery-free sensor node $v \in U \cup C$ is computed and determined. The energy status of battery-free sensor node $v \in U \cup C$ and its receivers in $R_{v}$ is estimated and considered in assigning transmission time for $v$. In this way, the broadcast schedule can make sure that each batteryfree sensor node $v \in U \cup C$ has enough energy to relay the broadcast data packet at its assigned 
transmission time and all receivers in $R_{v}$ have enough energy to receive the broadcast data packet. The correctness of the SBS algorithm is analyzed in detail by the following theorem.

THEOREM 2. The SBS algorithm produces correct broadcast schedules for BF-WSNs.

Proof. Let $S C H_{S B S}=\left\{\left\langle\{s\}, t_{1}\right\rangle,\left\langle s_{2}, t_{2}\right\rangle, \ldots,\left\langle s_{l}, t_{l}\right\rangle\right\}$ be the output of the SBS algorithm, and $\mathcal{T}$ be the tree generated by Step 1 of the Tran-Seq-Generator algorithm. From Step 3 of the TranTime-Assigner1 algorithm, $S C H_{S B S}$ can also be expressed by $S C H_{S B S}=\bigcup_{i=0}^{R} U T_{i} \cup C T_{i}$, where $R$ is the depth of $\mathcal{T}$. According to the Tran-Time-Assigner1 algorithm, we have $U=\{v \mid v \in S,\langle S, t\rangle \in$ $\left.\bigcup_{i=0}^{R} U T_{i}\right\}$ and $C=\left\{v \mid v \in S,\langle S, t\rangle \in \bigcup_{i=0}^{R} C T_{i}\right\}$. From Step 1 of the Tran-Time-Assigner1 algorithm, $R_{v}$ is the set of sensor nodes that receive the broadcast data packet sent by $v \in U \cup C$.

First, we need to prove that each battery-free sensor node $v \in U \cup C$ has enough energy to relay the broadcast data packet and all receivers in $R_{v}$ have enough energy to receive the broadcast data packet at the transmission time $t_{v}$ assigned by $S C H_{S B S}$. According to the definition of $t_{v}$ in the transmission time assignment of the SBS algorithm, we have

$$
t_{v} \geq \max \left\{t^{-}+\Delta, t_{0}+\max \left\{T_{u}\left(E_{f}\right) \mid u \in R_{v}\right\}\right\} .
$$

Therefore, $t_{v} \geq t_{0}+\max \left\{T_{u}\left(E_{f}\right) \mid u \in R_{v}\right\}$, which means all the battery-free sensor nodes in $R_{v}$ have harvested enough energy to receive the broadcast data packet. Besides, we have $t_{v} \geq t^{-}+\Delta$ according to Formula (2). By the definition of $t^{-}, v$ must have received the broadcast data packet before time $t^{-}+\Delta$. Since $v$ must have harvested of $E_{f}$ energy before it begins to receive the broadcast data packet, then $v$ must have enough energy to relay the broadcast data packet at time $t_{v}$.

Second, we need to prove that $S C H_{S B S}$ can result in that all the battery-free sensor nodes in the input BF-WSN except the source sensor node $s$ will receive the broadcast data packet initiated by $s$, i.e.,

$$
V \backslash\{s\}=\bigcup_{v \in U \cup C} R_{v} .
$$

$\forall u \in \bigcup_{v \in U \cup C} R_{v}$, sensor node $u \in V \backslash\{s\}$, since $s$ does not receive the broadcast data packet from any sensor node and thus is not in any $R_{v}$. Therefore, we have

$$
\bigcup_{v \in U \cup C} R_{v} \subseteq V \backslash\{s\}
$$

$\forall u \in V \backslash\{s\}$, we need to prove $u \in \bigcup_{v \in U \cup C} R_{v}$. From the construction procedure of $L S_{i}, U$ is the maximal independent set of $G(V, E)$. From the construction procedure of tree $\mathcal{T}$, sensor nodes in $C$ are the parents of the sensor nodes in $U$ on $\mathcal{T}$. Thus, $U \cup C$ is a connected dominating set of $G(V, E)$. Therefore, $\forall u \in V \backslash\{s\}$, which is a sensor node in some layer $L_{i}$, there is at least one neighbor of it in $U \cup C$, i.e., $N(u) \cap(U \cup C) \neq \emptyset$. Moreover, there is at least one sensor node of $N(u) \cap(U \cup C)$ in layer $L_{i-1}$. Let $N^{\prime}(u)=\left\{v \mid v \in N(u) \cap(U \cup C) \cap L_{i-1}\right\}$. According to the receiving set construction method in the SBS algorithm, $\forall u \in V \backslash\{s\}$, there must be a sensor node $v \in N^{\prime}(u)$ so that $u \in R_{v}$, i.e., $u \in \bigcup_{v \in N^{\prime}(u)} R_{v}$. Since $N^{\prime}(u) \subseteq U \cup C$ we have $u \in \bigcup_{v \in U \cup C} R_{v}$. Therefore,

$$
V \backslash\{s\} \subseteq \bigcup_{v \in U \cup C} R_{v}
$$

According to Formula (4) and Formula (5), we have proved that $V \backslash\{s\}=\bigcup_{v \in U \cup C} R_{v}$.

In conclusion, we have proved that the SBS algorithm produces correct broadcast schedules for BF-WSNs. 


\subsection{The Waiting Time Shortened Broadcast Scheduling Algorithm}

The Waiting Time Shortened Broadcast Scheduling (WTSBS) algorithm is designed for the BFWSNs where energy harvesting rates of battery-free sensor nodes are relatively low. In the SBS algorithm, a sensor node $v \in U \cup C$ will not transmit the broadcast data packet until all batteryfree sensor nodes in its receiving set $R_{v}$ have harvested $E_{f}$ energy. When battery-free sensor nodes in $R_{v}$ have low energy harvesting rates, the time consumption for them to harvest $E_{f}$ energy is large. Therefore, the waiting time of $v$ may be long and the broadcast latency of the broadcast schedule will increase accordingly.

The energy consumption for a battery-free sensor node to receive a broadcast data packet of $B$ bits is denoted by $\alpha E_{f}$ and the energy consumption for a battery-free sensor node to relay the broadcast data packet is denoted as $(1-\alpha) E_{f}$, where $0<\alpha<1$. Usually, $0<\alpha<\frac{1}{2}$, since the energy consumption for transmitting a data packet is larger than the energy consumption for receiving the same data packet [44].

The WTSBS algorithm shortens the waiting time of each battery-free sensor node in $U \cup C$ by taking the states and state transition of each battery-free sensor node during a broadcast into consideration in assigning transmission times. The states of a battery-free sensor node during a broadcast are charging state, receiving state and transmitting state. The state transition of batteryfree sensor node $v$ is as follows. First, $v$ is in the charging state and starts to harvest energy. If $v$ has harvested $\alpha E_{f}$ energy, then $v$ wakes up and gets ready to receive the broadcast data packet. Then, when a neighbor of $v$ transmits the broadcast data packet to $v, v$ changes to the receiving state and receives the broadcast data packet. After $v$ has received the broadcast data packet, its residual energy may be not enough for $v$ to relay the broadcast data packet. If so, then $v$ changes to the charging state again. Anyway, when $v$ has $(1-\alpha) E_{f}$ energy, it wakes up and transmits the broadcast data packet at the transmission time assigned to it, i.e., $v$ changes to the transmitting state.

The WTSBS algorithm consists of two phases, i.e., Transmission Sequence Generation and Transmission Time Assignment. The details are as follows.

3.2.1 Phase One: Transmission Sequence Generation. This phase is the same as the first phase of the SBS algorithm. The transmission sequence $\left\{L S_{i} \mid 0 \leq i \leq R\right\}$ is computed by Algorithm 1 .

3.2.2 Phase Two: Transmission Time Assignment. Based on the transmission sequence $\left\{L S_{i} \mid 0 \leq\right.$ $i \leq R\}$ computed by the first phase, the second phase assigns transmission times for sensor nodes in every element of $L S_{i}$ from $i=0$ to $R$. The second phase is named as Tran-Time-Assigner2, shown in Algorithm 4. There are three steps.

Step 1. Build the receiving set $R_{v}$ for each sensor node $v$ in each $U_{i j}\left(1 \leq j \leq u_{i}\right)$ or $C_{i j}(1 \leq j \leq$ $c_{i}$ ) of $L S_{i}$. This step is the same as Step 1 of the second phase in the SBS algorithm.

Step 2. Assign transmission times for sensor nodes in each element of $L S_{i}=\left(U_{i 1}, \ldots, U_{i u_{i}}\right.$, $\left.C_{i 1}, \ldots, C_{i c_{i}}\right)$.

At the transmission time of a battery-free sensor node $v$, all battery-free sensor nodes in $R_{v}$ must have harvested $\alpha E_{f}$ energy to receive the broadcast data packet. Besides, $v$ must have harvested $(1-\alpha) E_{f}$ energy to relay the broadcast data packet. The methods of assigning transmission times for sensor nodes in each element of $L S_{i}$ are shown as follows.

Step 2.1. Assign transmission time for each sensor node in $U_{i j}$ for $1 \leq j \leq u_{i}$ to generate a partial schedule $U T_{i}$. Let $t^{-}$be the latest transmission time that has already been assigned to a sensor node in the elements of $L S_{i-1}$.

For $\forall v$ in $U_{i j}$, we first initialize $t_{v}$ as

$$
\max \left\{t^{-}+\Delta, t_{0}+T_{v}\left(E_{f}\right), t_{0}+\max \left\{T_{u}\left(\alpha E_{f}\right) \mid u \in R_{v}\right\}\right\} .
$$




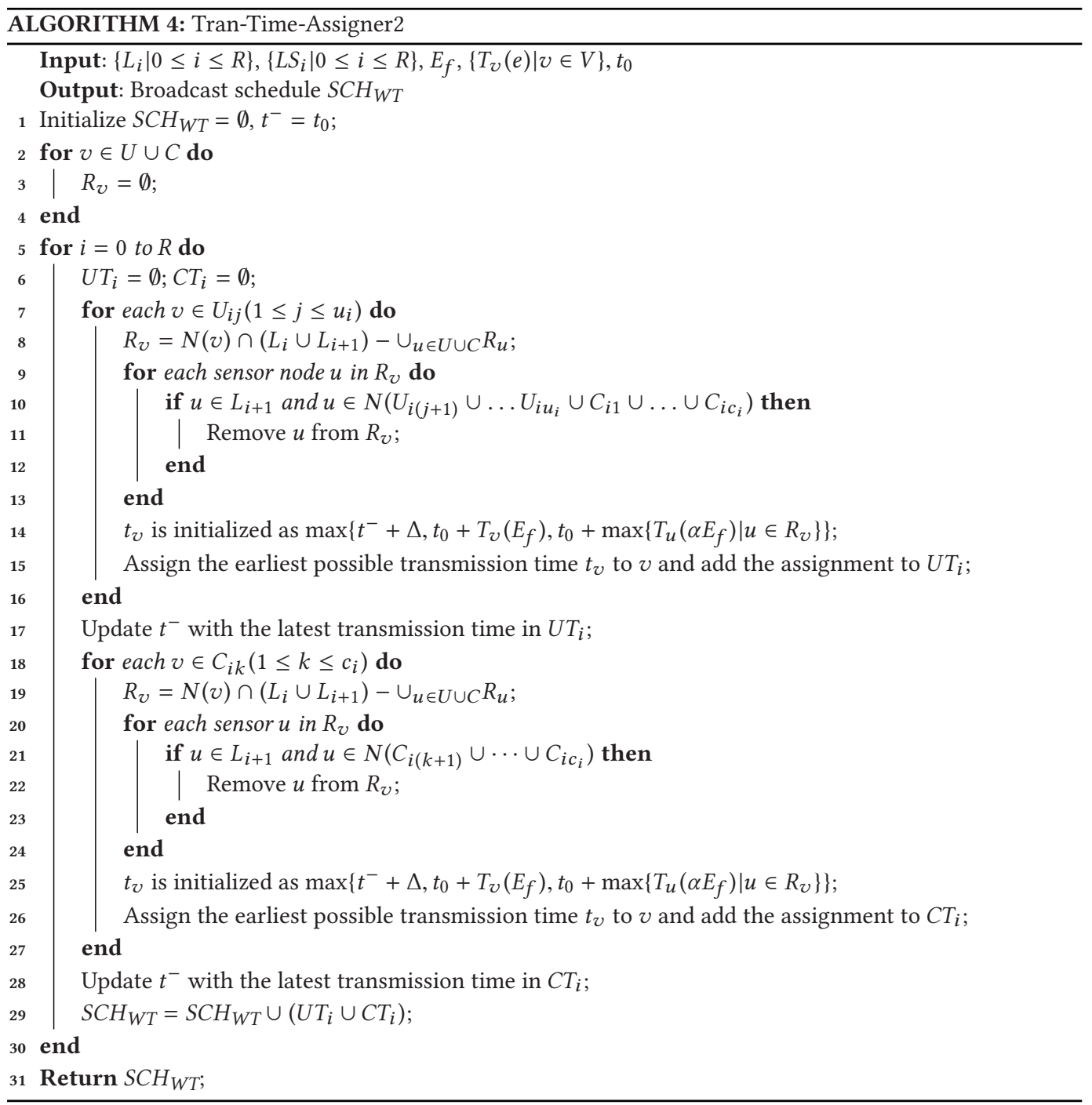

$T_{v}\left(E_{f}\right)$ is the time consumption for sensor node $v$ to harvest enough energy for transmitting and receiving the broadcast data packet and $T_{v}\left(\alpha E_{f}\right)$ is the time consumption for $v$ to harvest enough energy for receiving the broadcast data packet. $T_{v}\left(E_{f}\right)$ and $T_{v}\left(\alpha E_{f}\right)$ are estimated by $v$ through energy prediction methods and are collected at the initial time $t_{0}$. It will be proved that the energy stored in $v$ and sensor nodes in $R_{v}$ is enough for the communication between them at $t_{v}$ in Theorem 3. Thus, assign a transmission time to $v$ and add the assignment into $U T_{i}$ as follows.

1. If $t_{v}$ has not been assigned to other sensor nodes in partial schedule $U T_{i}$, then $t_{v}$ can be assigned to $v$ without collisions. Add $\left\langle\{v\}, t_{v}\right\rangle$ to $U T_{i}$.

2. If $t_{v}$ has already been assigned to some sensor nodes in $U T_{i}$, i.e. $\left\langle S, t_{v}\right\rangle \in U T_{i}$, but $v$ and sensor nodes in $S$ can transmit simultaneously without collisions, then update $\left\langle S, t_{v}\right\rangle \in U T_{i}$ with $\left\langle S \cup\{v\}, t_{v}\right\rangle$. 


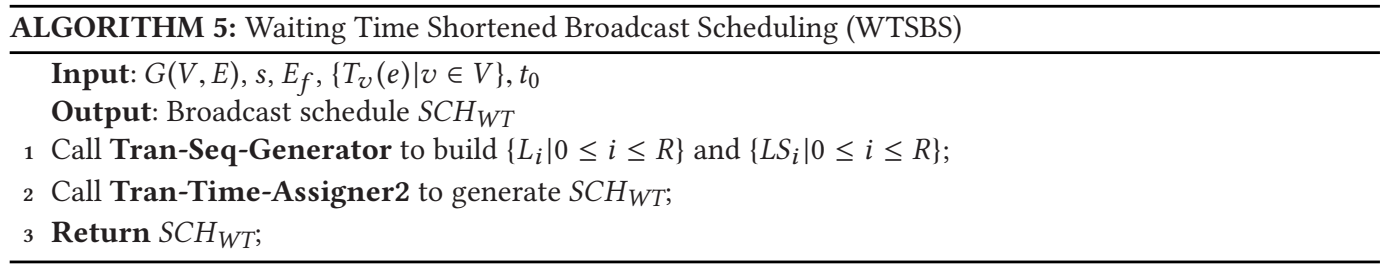

3. If $t_{v}$ has already been assigned to some sensor nodes in $U T_{i}$ and the concurrent transmissions of $v$ and one of these sensor nodes will cause collision, then the earliest transmission time for $v$ is $t_{v}+\Delta$. Increase $t_{v}$ by $\Delta$ and go to 1 .

Step 2.2. Assign transmission times for sensor nodes in $C_{i k}\left(1 \leq k \leq c_{i}\right)$ to generate another partial schedule $C T_{i}$. Let $t^{-}$be the latest transmission time assigned to sensor nodes in $U T_{i}$.

For each sensor node $v$ in $C_{i k}$, we first initialize $t_{v}$ as Formula (6). Then, assign a transmission time to $v$ and add the assignment into $C T_{i}$ as follows.

1. If $t_{v}$ has not been assigned to another sensor node in partial $C T_{i}$, then $t_{v}$ is assigned to $v$ and update $C T_{i}$ with $C T_{i}=C T_{i} \cup\left\{\left\langle\{v\}, t_{v}\right\rangle\right\}$.

2. If $t_{v}$ has been assigned to a sensor node set $S$ in $C T_{i}$, i.e., $\left\langle S, t_{v}\right\rangle \in C T_{i}$, but the concurrent transmissions of $v$ and all sensor nodes in $S$ are collision-free, then update $\left\langle S, t_{v}\right\rangle$ with $\left\langle S \cup\{v\}, t_{v}\right\rangle$.

3. If neither of the above two conditions is true, then the earliest transmission time can be assigned to $v$ is $t_{v}+\Delta$. Then, increase $t_{v}$ by $\Delta$ and go to 1 .

Step 3. Update the broadcast schedule $S C H_{W T}$ by $S C H_{W T}=S C H_{W T} \cup\left(U T_{i} \cup C T_{i}\right)$.

The WTSBS algorithm consists of two phases, Tran-Seq-Generator shown in Algorithm 1 and Tran-Time-Assigner2 shown in Algorithm 4. The pseudocode for the WTSBS algorithm is shown in Algorithm 5.

The WTSBS algorithm can generate collision-free broadcast schedules for BF-WSNs and can reduce broadcast latency when battery-free sensors have low energy harvesting rates. To verify the feasibility of the WTSBS algorithm for broadcast scheduling in BF-WSN, we need to prove the correctness. First, we prove that at the assigned transmission time $t_{v}$, sensor node $v \in U \cup C$ has enough energy for its transmission and all the sensor nodes in $R_{v}$ have enough energy for data receiving. This is shown in Theorem 3.

Theorem 3. At the assigned transmission time $t_{v}$ of sensor node $v, v$ has at least $(1-\alpha) E_{f}$ energy to transmit its received broadcast data packet, and all sensor nodes in $R_{v}$ have at least $\alpha E_{f}$ energy to receive the broadcast data packet transmitted by $v$.

Proof. The broadcast is initiated at time $t_{0}$ by the source sensor node $s$. At time $t_{0}$, not all sensor nodes have enough energy except $s$. We first prove that $v$ has at least $(1-\alpha) E_{f}$ energy at time $t_{v}$.

From the definition of $t_{v}, t_{v} \geq \max \left\{t^{-}+\Delta, t_{0}+T_{v}\left(E_{f}\right), t_{0}+\max \left\{T_{u}\left(\alpha E_{f}\right) \mid u \in R_{v}\right\}\right\}$. Therefore, we have $t_{v} \geq t^{-}+\Delta$. By the definition of $t^{-}$, $v$ must have received the broadcast data packet before time $t^{-}+\Delta$. If $v$ receives the broadcast data packet at time $t^{r}$, then $t^{r} \leq t^{-}+\Delta \leq t_{v}$ and $v$ turns to charging state after time $t^{r}+\Delta$. Let $t^{e}$ be the earliest time at which $v$ has harvested energy of $(1-\alpha) E_{f}$ and wakes up again. At time $t^{r}, v$ has harvested $E^{r}$ energy. After receiving the broadcast data packet at time $t^{r}+\Delta$, $v$ 's residual energy is $E^{r}-\alpha E_{f}$. The time consumption for $v$ to harvest 
energy from $E^{r}-\alpha E_{f}$ to $(1-\alpha) E_{f}$ is

$$
\begin{aligned}
t^{e}-\left(t^{r}+\Delta\right) & =T_{v}\left((1-\alpha) E_{f}\right)-T_{v}\left(E^{r}-\alpha E_{f}\right) \\
& \leq T_{v}\left(E_{f}\right)-T_{v}\left(E^{r}\right) \\
& \leq T_{v}\left(E_{f}\right)-T_{v}\left(E^{r}-\alpha E_{f}\right) \\
& =T_{v}\left(E_{f}\right)-\left(t^{r}+\Delta-t_{0}\right)
\end{aligned}
$$

The first inequality holds, because $E_{f} \geq(1-\alpha) E_{f}, E^{r} \geq E^{r}-\alpha E_{f}, E_{f}-E^{r}=(1-\alpha) E_{f}-\left(E^{r}-\right.$ $\left.\alpha E_{f}\right)$ and $T_{v}(e)$ is a convex function. Thus, we have $t_{0}+T_{v}\left(E_{f}\right) \geq t^{e}$ from Formula (7). From the definition of $t_{v}$, we have $t_{v} \geq t_{0}+T_{v}\left(E_{f}\right)$. Therefore, $t_{v} \geq t_{e}$. Since $v$ has harvested $(1-\alpha) E_{f}$ energy at time $t_{e}, v$ must have harvested more than $(1-\alpha) E_{f}$ energy at time $t_{v}$.

Now, we prove that all sensor nodes in $R_{v}$ have at least $\alpha E_{f}$ energy at time $t_{v}$. From the definition of $t_{v}, t_{v} \geq t_{0}+\max \left\{T_{u}\left(\alpha E_{f}\right) \mid u \in R_{v}\right\}$. Since every sensor node in $R_{v}$ must have harvested $\alpha E_{f}$ energy at time $t_{0}+\max \left\{T_{u}\left(\alpha E_{f}\right) \mid u \in R_{v}\right\}$, it has at least $\alpha E_{f}$ energy at time $t_{v}$.

Based on Theorem 3, the following theorem shows the correctness of the broadcast schedules produced the WTSBS algorithm.

THEOREM 4. The WTSBS algorithm produces correct broadcast schedules for BF-WSNs.

Proof. Let $S C H_{W T}=\left\{\left\langle\{s\}, t_{1}\right\rangle,\left\langle s_{2}, t_{2}\right\rangle, \ldots,\left\langle s_{l}, t_{l}\right\rangle\right\}$ be a broadcast schedule produced by the WTSBS algorithm. We have proved in Theorem 3 that every sensor node $v$ in $U \cup C$ of $S C H_{W T}$ has at least $(1-\alpha) E_{f}$ energy and all the sensor nodes in $R_{v}$ have at least $\alpha E_{f}$ energy at the transmission time $t_{v}$ assigned to $v$. Thus, each sensor node $v$ in $U \cup C$ can transmit the broadcast data packet and all the sensor nodes in $R_{v}$ can receive it at time $t_{v}$.

To prove the correctness of $S C H_{W T}$, i.e., $S C H_{W T}$ can result in that the broadcast data packet sent by $s$ can be received by all the sensor nodes in the input BF-WSN except $s$, we only need to prove that $V \backslash\{s\}=\bigcup_{v \in U \cup C} R_{v}$ in the following two stages:

(1) Since the source node $s$ does not receive the broadcast data packet from other sensor nodes, then $s$ is not in any receiving set $R_{v}$, where $v \in U \cup C$. Therefore, $\forall u \in \cup_{v \in U \cup C} R_{v}$, $u \in V \backslash\{s\}$. Then we have $\bigcup_{v \in U \cup C} R_{v} \subseteq V \backslash\{s\}$.

(2) We need to prove that $\forall u \in V \backslash\{s\}, u \in \bigcup_{v \in U \cup C} R_{v}$. According to the construction procedure of transmission sequences $L S_{i}(0 \leq i \leq R), U$ is the maximal independent set of $G(V, E)$. The sensor nodes in $C$ are the parents of the sensor nodes in $U$ in the breadthfirst searching tree $\mathcal{T}$. Thus, $U \cup C$ is a connected dominating set of $G(V, E)$. Therefore, $\forall u \in V \backslash\{s\}$, there is at least one neighbor of it in $U \cup C$, i.e., $N(u) \cap(U \cup C) \neq \emptyset$. Sensor node $u$ 's layer is denoted as $L_{i}$. According to the construction of the breadth-first searching tree, there is at least one sensor node of $N(u) \cap(U \cup C)$, which is the parent of $u$ in layer $L_{i-1}$. Let $N^{\prime}(u)=\left\{v \mid v \in N(u) \cap(U \cup C) \cap L_{i-1}\right\}$. According to the receiving set construction method in the WTSBS algorithm, $\forall u \in V \backslash\{s\}$, there must be a sensor node $v \in N^{\prime}(u)$ so that $u \in R_{v}$, i.e., $u \in \bigcup_{v \in N^{\prime}(u)} R_{v}$. Since $N^{\prime}(u) \subseteq U \cup C$, then we have $u \in \bigcup_{v \in U \cup C} R_{v}$. Therefore, we have proved that $V \backslash\{s\} \subseteq \bigcup_{v \in U \cup C} R_{v}$.

As a consequence, based on the above two stages, we have $V \backslash\{s\}=\bigcup_{v \in U \cup C} R_{v}$.

In summary, we have proved that the WTSBS algorithm can produce correct broadcast schedules for BF-WSNs. 


\subsection{The Enhanced Waiting Time Shortened Broadcast Scheduling Algorithm}

The Enhanced Waiting Time Shortened Broadcast Scheduling (EWTSBS) algorithm is designed for the BF-WSNs where there is a great difference in the energy harvesting rates of battery-free sensor nodes in the same layer. In the SBS algorithm and the WTSBS algorithm, a sensor node $v$ in layer $L_{i}$ only transmits the broadcast data packet to sensor nodes in layer $L_{i}$ and $L_{i+1}$. That is, $R_{v} \subset L_{i} \cup L_{i+1}$ if $v \in L_{i}$. When there is a great difference in the energy harvesting rates of batteryfree sensor nodes in the same layer, the following case may occur.

Sensor node $u \in L_{i+1}$ is in the receiving set $R_{v}$ of sensor node $v \in L_{i}$, but the energy harvesting rate of $u$ is much lower than that of other battery-free sensor nodes in $R_{v}$. As a consequence, the waiting time of $v$ will be long on account of $u$. In this case, $v$ becomes a bottleneck in reducing broadcast latency.

The EWTSBS algorithm breaks through this bottleneck by adopting the following strategy. Actually, it is better to add $u$ into the receiving set of some sensor node $w$ in layer $L_{j}(j>i)$ that has latter transmission time than $v$ if possible. In this way, the transmission time of $v$ can be earlier, since $v$ does not need to wait $u$ to wake up and receive the broadcast data packet from $v$. As a consequence, the broadcast latency of the broadcast schedule can be reduced.

The EWTSBS algorithm has two phases, i.e., Transmission Sequence Generation and Transmission Time Assignment. The details are as follows.

3.3.1 Phase One: Transmission Sequence Generation. This phase is the same as the first phase of the SBS algorithm and the WTSBS algorithm. Algorithm 1 is applied to generate the transmission sequence $\left\{L S_{i} \mid 0 \leq i \leq R\right\}$.

3.3.2 Phase Two: Transmission Time Assignment. The second phase of the EWTSBS algorithm is called Tran-Time-Assigner3, which is shown in Algorithm 6. Tran-Time-Assigner3 assigns transmission times for sensor nodes in each element of $\left\{L S_{i} \mid 0 \leq i \leq R\right\}$ by the following three steps.

Step 1. Build the receiving set for each sensor node $v$ in each $U_{i j}\left(1 \leq j \leq u_{i}\right)$ or $C_{i j}\left(1 \leq j \leq c_{i}\right)$ of $L S_{i}$. In this subsection, the receiving set of a sensor node $v$ is denoted as $W_{v}$ to distinguish it from the receiving set $R_{v}$ in the above two subsections. The construction of $W_{v} \mathrm{~s}$ is shown in Line 2 to Line 23 in Algorithm 6.

First, every receiving set is initialized as $\emptyset$. Then, for each sensor node $u$ in layer $L_{i}$ for $R \geq i \geq 1$, add $u$ to a proper receiving set $W_{v}$ as follows:

(1). (Lines 7-9) If $u \notin\left(\bigcup_{j=1}^{u_{i}} U_{i j}\right) \cup\left(\bigcup_{k=1}^{c_{i}} C_{i k}\right)$, which means $u$ does not need to transmit the broadcast data packet received by it to other sensor nodes, then $u$ is added to the receiving set $W_{v}$, where $v$ satisfies the following condition. If $h=\max \left\{p \mid\left(N(u) \cap\left(\bigcup_{j=1}^{u_{p}} U_{p j} \cup\right.\right.\right.$ $\left.\left.\bigcup_{k=1}^{c_{p}} C_{p k}\right)\right) \neq \emptyset$ and $\left.0 \leq p \leq R\right\}$ and the transmission sequence $L S_{h}=\left(l s_{1}, \ldots, l s_{m}\right)$, then randomly select $v$ in $l s_{j} \cap N(u)$, where $j=\max \left\{k \mid N(u) \cap l s_{k} \neq \emptyset\right.$ and $\left.1 \leq k \leq m\right\}$.

(2). (Lines 10-13) If $u \in \bigcup_{k=1}^{c_{i}} C_{i k}$ and $N(u) \cap\left(\bigcup_{j=1}^{u_{i}} U_{i j}\right) \neq \emptyset$, which means $u$ has to receive the broadcast data packet from sensor nodes in $\bigcup_{j=1}^{u_{i}} U_{i j}, u$ is added to the receiving set $W_{v}$, where $v=\arg \max _{o \in N(u)}\left\{j \mid o \in U_{i j}\right.$ and $\left.1 \leq j \leq u_{i}\right\}$.

(3). (Lines 14-17) If $u \in \bigcup_{k=1}^{c_{i}} C_{i k}$ but $N(u) \cap\left(\bigcup_{j=1}^{u_{i}} U_{i j}\right)=\emptyset$, then it means $u$ receives the broadcast data packet from a neighbor in layer $L_{i-1}$. Assuming that the transmission sequence of layer $L_{i-1}$ is $L S_{i-1}=\left(l_{1}, \ldots, l s_{m}\right), u$ is added to the receiving set $W_{v}$, where $v$ is in $l s_{j} \cap N(u)$ and $j=\max \left\{k \mid N(u) \cap l s_{k} \neq \emptyset\right.$ and $\left.1 \leq k \leq m\right\}$.

(4). (Lines 18-20) If $u \in \cup_{j=1}^{u_{i}} U_{i j}$, which means $u$ has to receive the broadcast data packet before all sensor nodes in all the elements of $L S_{i-1}$ complete their transmissions, then $u$ is added to the receiving set $W_{v}$, where $v=\arg \max _{o \in N(u)}\left\{j \mid o \in C_{(i-1) j}\right.$ and $\left.1 \leq j \leq c_{i-1}\right\}$. 
Step 2. Assign transmission times for sensor nodes in each element of $L S_{i}=\left(U_{i 1}, \ldots, U_{i u_{i}}\right.$, $\left.C_{i 1}, \ldots, C_{i c_{i}}\right)$ based on the receiving sets constructed by Step 1. This step is the same as Step 2 of the second phase in the WTSBS algorithm.

Step 3. Update the broadcast schedule $S C H_{E W T}$ by $S C H_{E W T}=S C H_{E W T} \cup\left(U T_{i} \cup C T_{i}\right)$.

The pseudocode of the EWTSBS algorithm is shown in Algorithm 7.

The EWTSBS algorithm can generate collision-free broadcast schedule for BF-WSNs and can reduce the broadcast latency of the broadcast schedule when there is a great difference in energy harvesting rates of battery-free sensor nodes in the same layers. The correctness of the EWTSBS algorithm is analyzed in details by the following theorem.

THEOREM 5. The EWTSBS algorithm produces correct broadcast schedules for BF-WSNs.

Proof. Let $S C H_{E W T}=\left\{\left\langle\{s\}, t_{1}\right\rangle,\left\langle s_{2}, t_{2}\right\rangle, \ldots,\left\langle s_{l}, t_{l}\right\rangle\right\}$ be the broadcast schedule produced by the EWTSBS algorithm. As discussed in the proof of Theorem 2, $S C H_{E W T}$ can also be expressed by $S C H_{E W T}=\bigcup_{i=0}^{R} U T_{i} \cup C T_{i}$. According to the Tran-Time-Assigner3 algorithm, we have $U=\{v \mid v \in$ $\left.S,\langle S, t\rangle \in \bigcup_{i=0}^{R} U T_{i}\right\}$ and $C=\left\{v \mid v \in S,\langle S, t\rangle \in \bigcup_{i=0}^{R} C T_{i}\right\}$.

First, we need to prove that at the transmission time $t_{v}$ of sensor node $v$ assigned by the EWTSBS algorithm, $v$ has at least $(1-\alpha) E_{f}$ energy and all the sensor nodes in its receiving set $W_{v}$ have at least $\alpha E_{f}$ energy. This proof is similar with that of Theorem 3.

Second, we need to prove that $S C H_{E W T}$ can result in that all battery-free sensor nodes in the input BF-WSN except the source sensor node $s$ will receive the broadcast data packet initialized by $s$. From Step 1 of the Tran-Time-Assigner3 algorithm, $W_{v}$ is the set of sensor nodes that receive the broadcast data packet sent by $v \in U \cup C$. Thus, we need to prove

$$
V \backslash\{s\}=\bigcup_{v \in U \cup C} W_{v} .
$$

Since the source sensor node $s$ does not receive the broadcast data packet from other sensor nodes, $s$ is not in any receiving set $W_{v}$, where $v \in U \cup C$. Therefore, $\forall u \in \cup_{v \in U \cup C} W_{v}, u$ satisfies that $u \in V \backslash\{s\}$. Thus,

$$
\bigcup_{v \in U \cup C} W_{v} \subseteq V \backslash\{s\}
$$

Next, we need to prove that $\forall u \in V \backslash\{s\}, u \in \bigcup_{v \in U \cup C} W_{v}$. According to the method of constructing the receiving set in the EWTSBS algorithm, $\forall u \in V \backslash\{s\}, u$ is added to a proper receiving set $W_{v}$, where $v \in U \cup C$. Then, $\forall u \in V \backslash\{s\}, u \in \cup_{v \in U \cup C} W_{v}$. Therefore,

$$
V \backslash\{s\} \subseteq \bigcup_{v \in U \cup C} W_{v}
$$

In summary, according to Formula (9) and Formula (10), we have $V \backslash\{s\}=\bigcup_{v \in U \cup C} W_{v}$.

In conclusion, we have proved that the EWTSBS algorithm produces correct broadcast schedules for BF-WSNs.

\section{ANALYSIS OF THE ALGORITHMS}

In this section, we analyze the SBS algorithm, the WTSBS algorithm and the EWTSBS algorithm theoretically. First, the computation complexity of the broadcast schedules produced by the proposed algorithms are proved. Furthermore, the upper bound and approximation ratio of the broadcast latency caused by the broadcast schedules produced by the SBS algorithm, the WTSBS algorithm and the EWTSBS algorithm are analyzed. 


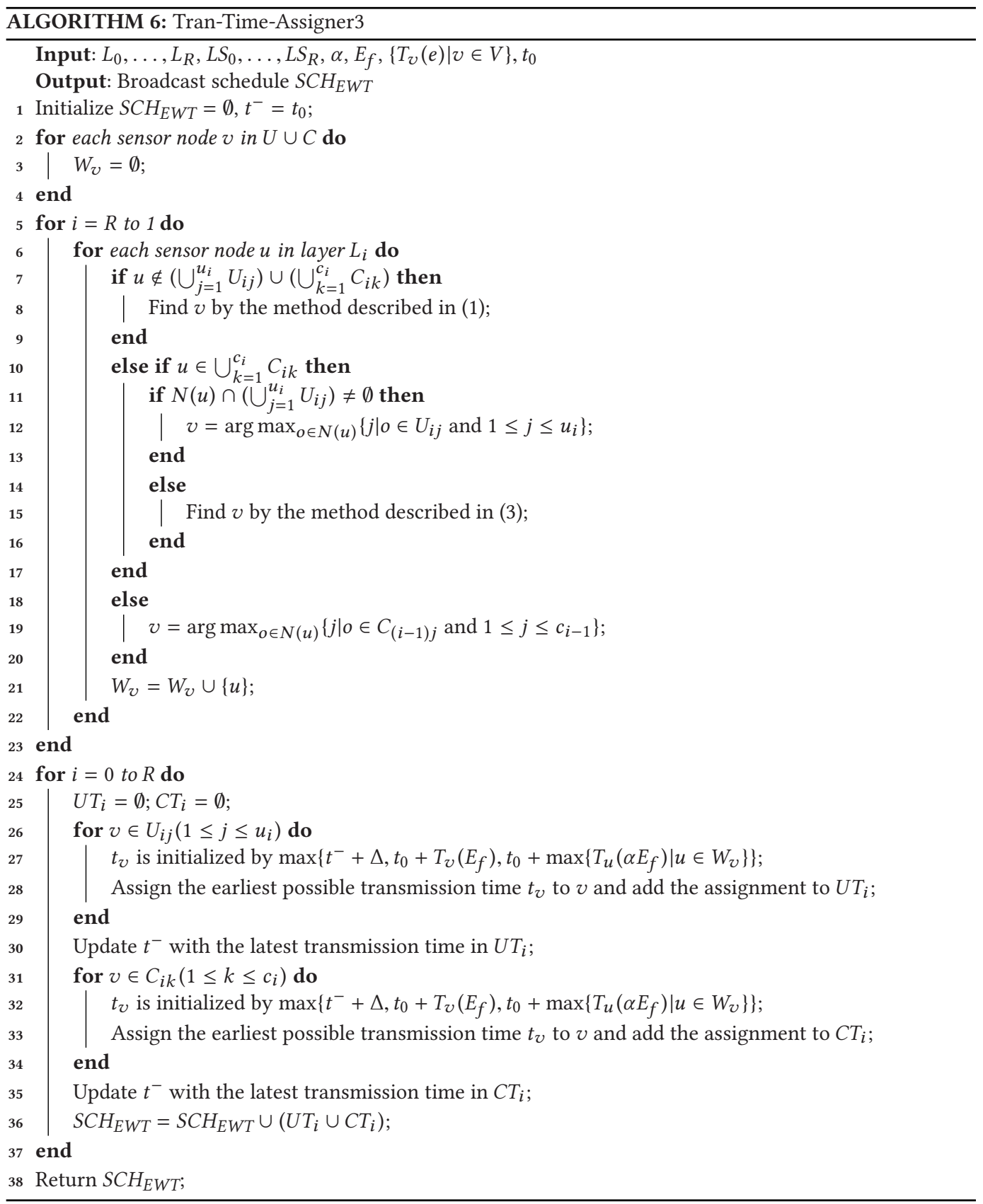

\subsection{Analysis of the SBS Algorithm}

4.1.1 The Computation Complexity of the SBS Algorithm. The computation complexity of the SBS algorithm is $O\left(n^{3}\right)$, which is analyzed as follows. The computation complexity of building the breadth-first searching tree for $G(V, E)$ in Step 1 of phase one is $O\left(n^{2}\right)$. The computation complexity of calculating the maximal independent set $U$ and set $C$ in Step 2 of phase one is $O\left(n^{2}\right)$. The 


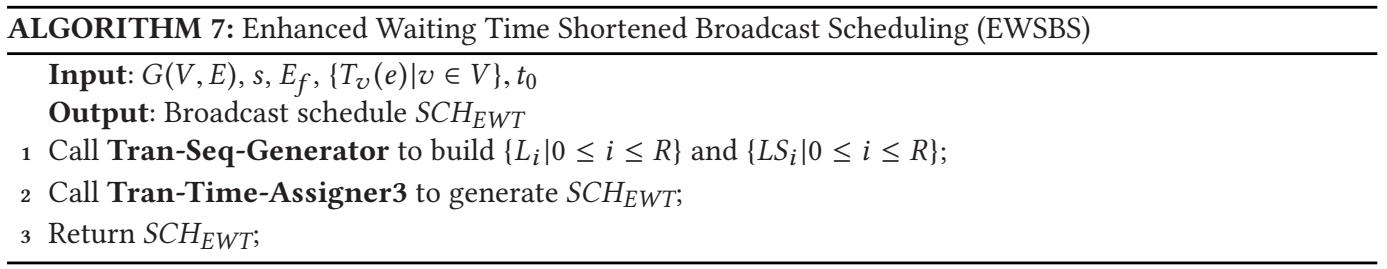

computation complexity of dividing set $U$ and $C$ in Step 3 of phase one is $O\left(n^{2}\right)$. Therefore, the computation complexity of the first phase, Transmission Sequence Generation, is $O\left(n^{2}\right)$. The computation complexity of constructing the receiving set $R_{v}$ for each sensor node in $U \cup C$ is $O\left(\mathrm{deg}^{2}\right)$, where deg is the maximum degree of all sensor nodes in graph $G(V, E)$. Besides, the computation complexity of assigning collision-free transmission time for each sensor node in $U \cup C$ is $O(n)$. Therefore, the computation complexity of the second phase, Transmission Time Assignment, is $O\left(n^{3}\right)$. In conclusion, the computation complexity of the SBS algorithm is $O\left(n^{3}\right)$.

\subsubsection{The Upper Bound and Approximation Ratio of the SBS Algorithm.}

Theorem 6. The broadcast latency of the broadcast schedule $S C H_{S B S}$ produced by the SBS algorithm is at most $16 R-15+T_{\max }$.

Proof. $T_{\text {max }}$ is defined as $\max \left\{T_{v}\left(E_{f}\right) \mid v \in V\right\}$. Since battery-free sensor nodes can harvest energy from their ambient environment simultaneously without interfering each other, all batteryfree sensor nodes have harvested $E_{f}$ energy at time $t_{0}+\max \left\{T_{v}\left(E_{f}\right) \mid v \in V\right\}=t_{0}+T_{\max }$, where $t_{0}$ is the initial time of the broadcast and $E_{f}$ is the energy consumption for a battery-free sensor node to receive and relay the broadcast data packet.

The worst case is that node $v$ with the maximum time consumption to harvest $E_{f}$ amount of energy is in layer $L_{1}$, i.e., $T_{v}\left(E_{f}\right)=T_{\max }=\max \left\{T_{u}\left(E_{f}\right) \mid u \in V\right\}$ and $v \in L_{1}$. Then the source sensor node has to transmit the broadcast data packet at time $t_{0}+T_{\max }$ according to the SBS algorithm. Then all battery-free sensor nodes have harvested at least $E_{f}$ amount of energy at time $t_{0}+T_{\max }$. Finally, the SBS algorithm degrades into the algorithm in Reference [25] in the remaining layers. The broadcast latency of scheduling the transmission sequence $\left\{L S_{i} \mid 0 \leq i \leq R\right\}$ is at most $16 R-15$ when every sensor node in the input BF-WSN has $E_{f}$ energy in its capacitor according to Reference [25]. Therefore, the maximum broadcast latency of the SBS algorithm is $16 R-15+T_{\max }$.

We use $T(S B S)$ to denote the broadcast latency of the broadcast schedule $S C H_{S B S}$ produced by the SBS algorithm, and $T(O P T)$ to denote the minimum broadcast latency of the optimal broadcast schedule $S C H_{O P T}$.

THEOREM 7. The broadcast latency of the optimal broadcast schedule $S C H_{O P T}$ is more than $2 T_{\text {min }}+$ $R$, where $T_{\min }=\min \left\{T_{v}\left(\frac{E_{f}}{2}\right) \mid v \in V\right\}$.

Proof. In the optimal broadcast schedule, all batter-free sensor nodes must have $\alpha E_{f}$ energy for receiving the broadcast data packet and then have $(1-\alpha) E_{f}$ energy for relaying the broadcast data packet, where $0<\alpha<1$. Since $T_{v}(e)$ is a non-decreasing and convex function [5], the ideal situation for a battery-free sensor node $u \in V$ is that $u$ receives the broadcast data packet at time $t_{0}+T_{u}\left(\alpha E_{f}\right)$ and relays the broadcast data packet at time $t_{0}+T_{u}\left(\alpha E_{f}\right)+T_{u}\left((1-\alpha) E_{f}\right)$. Since $R$ $(R \geq 1)$ is the depth of the breadth-first searching tree rooted at the source sensor node, there must be a sensor node $u \in R_{w}$, which is $R$ hops away from the source sensor node. Therefore, the earliest time for $u$ to receive the broadcast data packet is $t_{0}+T_{w}\left(\alpha E_{f}\right)+T_{w}\left((1-\alpha) E_{f}\right)+R$. As a consequence, the lower bound of the broadcast latency $T(O P T)$ of the optimal broadcast schedule 
$S C H_{O P T}$ is

$$
\begin{aligned}
T(O P T) & \geq\left(t_{0}+T_{w}\left(\alpha E_{f}\right)+T_{w}\left((1-\alpha) E_{f}\right)+R\right)-t_{0} \\
& =T_{w}\left(\alpha E_{f}\right)+T_{w}\left((1-\alpha) E_{f}\right)+R \\
& \geq 2 T_{w}\left(\frac{E_{f}}{2}\right)+R \\
& \geq 2 \min \left\{T_{v}\left(\frac{E_{f}}{2}\right) \mid v \in V\right\}+R \\
& =2 T_{\text {min }}+R .
\end{aligned}
$$

The second inequality holds, because $T_{v}(e)$ is a non-decreasing and convex function.

THEOREM 8. The approximation ratio of the SBS algorithm is $\frac{T(S B S)}{T(O P T)} \leq \frac{16 R-15+T_{\max }}{2 T_{\min }+R}$, where $T_{\max }=$ $\max \left\{T_{v}\left(E_{f}\right) \mid v \in V\right\}$ and $T_{\min }=\min \left\{T_{v}\left(\frac{E_{f}}{2}\right) \mid v \in V\right\}$.

PROOF. The lower bound of the broadcast latency of the optimal broadcast schedule $S C H_{O P T}$ is $T(O P T) \geq 2 T_{\min }+R$ proved in Theorem 7 . We have also proved that the broadcast latency of the broadcast schedule $S C H_{S B S}$ produced by the SBS algorithm is $T(S B S) \leq 16 R-15+T_{\max }$ in Theorem 6. As a consequence, we have $\frac{T(S B S)}{T(O P T)} \leq \frac{16 R-15+T_{\max }}{2 T_{\min }+R}$.

\subsection{Analysis of the WTSBS Algorithm}

4.2.1 The Computation Complexity of the WTSBS Algorithm. The computation complexity of the WTSBS algorithm is $O\left(n^{3}\right)$, which is analyzed as follows. The computation complexity of the first phase, Transmission Sequence Generation, is $O\left(n^{2}\right)$, which is the same as the first phase of the SBS algorithm. For the second phase, Transmission Time Assignment, the computation complexity is $O\left(n^{3}\right)$. First, the computation complexity of building the receiving set for a sensor node in $U \cup C$ is $O\left(\mathrm{deg}^{2}\right)$, where $d e g \leq n$. Then, the computation complexity of assigning a transmission time for each sensor node in $U \cup C$ is $O(n)$. And the number of sensor nodes in $U \cup C$ is bounded by $n$. Thus, the computation complexity of the second phase is $O\left(n^{3}\right)$. In conclusion, the computation complexity of the WTSBS algorithm is $O\left(n^{3}\right)$.

\subsubsection{The Upper Bound and Approximation Ratio of the WTSBS Algorithm.}

THEOREM 9. The upper bound of the broadcast latency of the broadcast schedule $S C H_{W T}$ produced by the WTSBS algorithm is $16 R-15+T_{\max }$.

Proof. Assume that the broadcast is initiated by the source sensor node $s$ at time $t_{0}$ in $\mathrm{SCH}_{W T}$. The time consumption for all battery-free sensor nodes to harvest $E_{f}$ energy from their environment is no more than $\max \left\{T_{v}\left(E_{f}\right) \mid v \in V\right\}$, which is denoted as $T_{\text {max }}$. Since each node can harvest energy from its ambient environment without affecting the others, every node in the input BFWSN must have $E_{f}$ energy in its capacitor at time $t_{0}+\max \left\{T_{v}\left(E_{f}\right) \mid v \in V\right\}=t_{0}+T_{\max }$.

The worst case of the WTSBS algorithm is that the battery-free sensor node with the maximum time consumption for harvesting $\alpha E_{f}$ energy is in layer $L_{1}$. Then, the source sensor node $s$ will transmit the broadcast data packet at time $t_{0}+\max \left\{T_{v}\left(\alpha E_{f}\right) \mid v \in L_{1}\right\}$, which is earlier than $t_{0}+T_{\max }$. Therefore, the broadcast latency of the broadcast schedule produced by the WTSBS algorithm is no more than the worst case of the SBS algorithm. Thus, the upper bound of the broadcast latency of the broadcast schedule $S C H_{W T}$ produced by the WTSBS algorithm is the same as that of the SBS algorithm, which is $16 R-15+T_{\max }$. 
We use $T(W T)$ to denote the broadcast latency of the broadcast schedule $S C H_{W T}$ produced by the WTSBS algorithm, and $T(O P T)$ to denote the minimum broadcast latency of the optimal broadcast schedule $S C H_{O P T}$.

THEOREM 10. The approximation ratio of the WTSBS algorithm is $\frac{T(W T)}{T(O P T)} \leq \frac{16 R-15+T_{\max }}{2 T_{\min }+R}$, where $T_{\text {max }}=\max \left\{T_{v}\left(E_{f}\right) \mid v \in V\right\}$ and $T_{\text {min }}=\min \left\{T_{v}\left(\frac{E_{f}}{2}\right) \mid v \in V\right\}$.

Proof. We have proved in Theorem 7 that the broadcast latency of the optimal broadcast schedule $S C H_{O P T}$ is more than $2 T_{\min }+R$, where $T_{\min }=\min \left\{T_{v}\left(\frac{E_{f}}{2}\right) \mid v \in V\right\}$. The broadcast latency of the broadcast schedule $S C H_{W T}$ produced by the WTSBS algorithm is proved to be less than $16 R-15+T_{\max }$ in Theorem 9. As a consequence, the approximation ratio of the WTSBS algorithm is $\frac{T(W T)}{T(O P T)} \leq \frac{16 R-15+T_{\max }}{2 T_{\min }+R}$.

\subsection{Analysis of the EWTSBS Algorithm}

4.3.1 The Computation Complexity of the EWTSBS Algorithm. The computation complexity of the EWTSBS algorithm is $O\left(n^{2}\right)$. First, the computation complexity of the first phase, Transmission Sequence Generation, is the same as that of the SBS algorithm, i.e., $O\left(n^{2}\right)$. The computation complexity of adding each sensor node in $V$ to a waiting set $W_{v}$ of sensor node $v$ is $O(d e g)$, where $\mathrm{deg}$ is the maximum degree of all sensor nodes in $G(V, E)$. Besides, the computation complexity of assigning collision-free transmission time for each sensor node in $U \cup C$ is $O(n)$. Therefore, the computation complexity of the second phase, Transmission Time Assignment, is $O\left(n^{2}\right)$. In conclusion, the computation complexity of the EWTSBS algorithm is $O\left(n^{2}\right)$.

\subsubsection{The Upper Bound and Approximation Ratio of the EWTSBS Algorithm.}

THEOREM 11. The broadcast latency of the broadcast schedule $S C H_{E W T}$ produced by the EWTSBS algorithm is no more than $16 R-15+T_{\max }$.

Proof. The initial time of the broadcast is $t_{0}$. The source sensor node $s$ can transmit the broadcast data packet when all the nodes in $R_{s}$ have harvested $\alpha E_{f}$ energy. Since all batteryfree sensor node can harvest energy from their ambient environment and they do not affect each other, the source sensor node $s$ must have transmitted the broadcast data packet at time $t_{0}+\max \left\{T_{v}\left(\alpha E_{f}\right) \mid v \in V\right\}$ in the broadcast schedule produced by the EWTSBS algorithm. Every node in the input BF-WSN must have $E_{f}$ energy in its capacitor at time $t_{0}+\max \left\{T_{v}\left(E_{f}\right) \mid v \in V\right\}=$ $t_{0}+T_{\text {max }}$. We have $t_{0}+\max \left\{T_{v}\left(\alpha E_{f}\right) \mid v \in L_{1}\right\} \leq t_{0}+T_{\max }$. Moreover, the EWTSBS algorithm optimizes the method of the receiving set construction, which reduces the waiting time of some nodes. Therefore, the broadcast latency of the broadcast schedule produced by the EWTSBS algorithm is no more than the worst case of the SBS algorithm. Thus, the upper bound of the broadcast latency of the broadcast schedule $S C H_{E W T}$ produced by the EWTSBS algorithm is the same as that of the SBS algorithm, which is $16 R-15+T_{\max }$.

We use $T(E W T)$ to denote the broadcast latency of the broadcast schedule $S C H_{E W T}$ produced by the EWTSBS algorithm, and $T(O P T)$ to denote the minimum broadcast latency of the optimal broadcast schedule $S C H_{O P T}$.

THEOREM 12. The approximation ratio of the EWTSBS algorithm is $\frac{T(E W T)}{T(O P T)} \leq \frac{16 R-15+T_{\max }}{2 T_{\min }+R}$, where $T_{\text {max }}=\max \left\{T_{v}\left(E_{f}\right) \mid v \in V\right\}$ and $T_{\text {min }}=\min \left\{T_{v}\left(\frac{E_{f}}{2}\right) \mid v \in V\right\}$.

Proof. We have proved in Theorem 7 that the lower bound of the broadcast latency of the optimal broadcast schedule $S C H_{O P T}$ is $2 T_{\text {min }}+R$, where $T_{\text {min }}=\min \left\{T_{v}\left(\frac{E_{f}}{2}\right) \mid v \in V\right\}$. In Theorem 11, we 
have proved that the upper bound of the broadcast latency of the broadcast schedule $S C H_{E W T}$ produced by the EWTSBS algorithm is $16 R-15+T_{\max }$. Thus, the approximation ratio of the EWTSBS algorithm is $\frac{T(E W T)}{T(O P T)} \leq \frac{16 R-15+T_{\max }}{2 T_{\min }+R}$.

\section{BROADCAST SCHEDULING UNDER PHYSICAL INTERFERENCE MODEL}

In this section, we discuss the broadcast scheduling for BF-WSNs under the physical interference model, which is more realistic but more complicated than the protocol interference model. The transmission scheduling for traditional WSNs under the physical interference model has been studied in References [24, 42, 51]. However, these works are not suitable for BF-WSNs. The reasons are similar to the reasons for the existing algorithms under protocol interference model, which are analyzed in Section 1.

As described in Section 2, a node $v_{j}$ can receive the data packet transmitted by $v_{i}$ if the SINR value between them is greater than a threshold, as shown in Formula (1). Therefore, the maximum transmission range of a sensor node is $\left(\frac{P}{N_{0} \beta}\right)^{1 / \alpha}$ when no other simultaneous transmissions exist. Therefore, the transmission range of all the nodes in a BF-WSN is denoted as $r_{s}=\left(\frac{P}{c N_{0} \beta}\right)^{1 / \alpha}$, where $c$ is a constant and $c>1$. The network topology of a BF-WSN is denoted by $G(V, E)$, where $\left(v_{i}, v_{j}\right) \in E$ if and only if $\operatorname{dist}\left(v_{i}, v_{j}\right) \leq r_{s}$. The neighbor set of $v_{i}$ is denoted as $N\left(v_{i}\right)$, where $v_{j} \in N\left(v_{i}\right)$ if $\operatorname{dist}\left(v_{i}, v_{j}\right) \in E$.

The broadcast scheduling for BF-WSNs under the physical interference model can be carried out in the following four steps.

Step 1. Divide all battery-free sensor nodes into layers according to $G(V, E)$. Firstly, build a breadth-first searching tree $\mathcal{T}$ rooted at $s$ on the topology graph $G(V, E) . R$ is the depth of $\mathcal{T}$. A sensor node $v_{i}$ is in layer $L_{j}$ if the number of hops from $v_{i}$ to $s$ is $j$ on tree $\mathcal{T}$. Therefore, all the nodes are divided into $R+1$ layers $L_{0}, L_{1}, \ldots, L_{R}$, where $L_{0}=\{s\}$.

Step 2. Generate transmission sequence for the BF-WSN. First, obtain the maximal independent set $U$ of $G(V, E)$ and $U$ can be divided into $U_{0}, U_{1}, \ldots, U_{R}$ such that $U_{i} \subseteq L_{i}$ for $0 \leq i \leq R$. Then, compute $C=\{u \mid u$ is the parent of $v$ in $\mathcal{T}$ and $v \in U\}$ and $C$ is also divided into $C_{0}, C_{1}, \ldots, C_{R}$ such that $C_{i} \subseteq L_{i}$ for $0 \leq i \leq R$. Since we have not computed the receiving set for each sensor node in $U \cup C$, we cannot compute set $U_{i k} \subset U_{i}$ or $C_{i l} \subset C_{i}$ where the nodes in $U_{i k}$ or $C_{i l}$ can transmit simultaneously without collisions under physical interference model. Therefore, the transmission sequence is $\left\{L S_{i} \mid 0 \leq i \leq R\right\}$, where $L S_{i}=\left\{U_{i}, C_{i}\right\}$.

Step 3. Compute the receiving set of each battery-free sensor node in $U \cup C$. First, $\forall v \in U \cup C$, the receiving set is $W_{v}=\emptyset$. Then, for each sensor node $u$ in layer $L_{i}$ for $R \geq i \geq 1$, add $u$ to a proper receiving set $W_{v}$ as follows.

1. If $u \notin U \cup C$, which means $u$ does not need to transmit the received broadcast data packet to other nodes, then $u$ is added to the receiving set $W_{v}$, where $v$ satisfies the following condition. Let $j=\max \left\{i \mid\left(N(u) \cap\left(U_{i} \cup C_{i}\right) \neq \emptyset\right.\right.$ and $\left.0 \leq i \leq R\right\}$. If $N(u) \cap C_{j} \neq \emptyset$, then randomly select a node $v \in N(u) \cap C_{j}$ and add $u$ to $W_{v}$. If $N(u) \cap C_{j}=\emptyset$, then randomly select a node $v \in N(u) \cap U_{j}$ and add $u$ to $W_{v}$.

2. If $u \in C_{i}$ and $N(u) \cap U_{i} \neq \emptyset$, which means $u$ can receive the broadcast data packet from the nodes in $U_{i}$, then randomly select a node $v \in U_{i}$ and add $u$ to $W_{v}$.

3. If $u \in C_{i}$ but $N(u) \cap U_{i}=\emptyset$, which means $u$ receives the broadcast data packet from a neighbor in layer $L_{i-1}$. If $N(u) \cap C_{i-1} \neq \emptyset$, then randomly select a node $v \in N(u) \cap C_{i-1}$ and add $u$ to $W_{v}$. If $N(u) \cap C_{i-1}=\emptyset$, then randomly select a node $v \in N(u) \cap U_{i-1}$ and add $u$ to $W_{v}$.

4. If $u \in U_{i}$, which means $u$ has to receive the broadcast data packet before all the nodes in all the elements of $L S_{i-1}$ complete their transmissions, then randomly select a node $v \in C_{i-1}$ and add $u$ to $W_{v}$. 
Step 4. For $0 \leq i \leq R$, assign transmission times for the nodes in each element of $L S_{i}=\left\{U_{i}, C_{i}\right\}$.

Step 4.1. Assign transmission time for each sensor node in $U_{i}$ to generate a partial schedule $U T_{i}$. Let $t^{-}$be the latest transmission time that has already been assigned to a node in the elements of $L S_{i-1}$.

For $\forall v$ in $U_{i}$, we first initialize $t_{v}$ as

$$
\max \left\{t^{-}+\Delta, t_{0}+T_{v}\left(E_{f}\right), t_{0}+\max \left\{T_{u}\left(\alpha E_{f}\right) \mid u \in W_{v}\right\}\right\} .
$$

Similarly, each battery-free sensor node $v$ estimated $T_{v}\left(E_{f}\right)$ and $T_{v}\left(\alpha E_{f}\right)$ by itself through energy prediction methods, where $T_{v}\left(E_{f}\right)$ presents the energy consumption for $v$ to harvest enough energy for transmitting and receiving the broadcast data packet and $T_{v}\left(\alpha E_{f}\right)$ presents the energy consumption for $v$ to harvest enough energy for receiving the broadcast data packet. The $T_{v}\left(E_{f}\right)$ and $T_{v}\left(\alpha E_{f}\right)$ are collected at the initial time $t_{0}$. Therefore, the sensor node $v$ and its receivers have accumulated enough energy for their transmission and reception at time $t_{v}$. Thus, assign a transmission time to $v$ and add the assignment into $U T_{i}$ as follows.

1. If $t_{v}$ has not been assigned to other nodes in $U T_{i}$, then $t_{v}$ can be assigned to $v$ without collisions. Add $\left\langle\{v\}, t_{v}\right\rangle$ to $U T_{i}$.

2. If $t_{v}$ has already been assigned to some nodes in $U T_{i}$, i.e., $\left\langle S, t_{v}\right\rangle \in U T_{i}$, then the SINR values of all the receivers at time $t_{v}$ including the nodes in $W_{v}$ should be recalculated.

(a) If the SINR values of all the receivers including the nodes in $W_{v}$ are all greater than $\beta$, meaning the transmissions of $v$ and the nodes in $S$ are collision-free under the physical interference model, then update $\left\langle S, t_{v}\right\rangle \in U T_{i}$ with $\left\langle S \cup\{v\}, t_{v}\right\rangle$.

(b) If any SINR value of the receivers including the nodes in $W_{v}$ is less than $\beta$, which means the concurrent transmissions of $v$ and at least one node in $S$ will cause collision, then the earliest transmission time for $v$ is $t_{v}+\Delta$. Increase $t_{v}$ by $\Delta$ and go to 1 .

Step 4.2. Assign transmission times for the nodes in $C_{i}$ to generate another partial schedule $C T_{i}$. Let $t^{-}$be the latest transmission time that has already been assigned to the nodes in $U T_{i}$.

For each node $v$ in $C_{i}$, we first initialize $t_{v}$ as Formula (12). Then, assign a transmission time to $v$ and add the assignment into $C T_{i}$ as follows.

1. If $t_{v}$ has not been assigned to other nodes in $C T_{i}$, then add $\left\langle\{v\}, t_{v}\right\rangle$ to $C T_{i}$.

2. If $t_{v}$ has already been assigned to some nodes in $C T_{i}$, i.e., $\left\langle S, t_{v}\right\rangle \in C T_{i}$, the SINR values of all the receivers at time $t_{v}$ including the nodes in $W_{v}$ should be recalculated.

(a) If the SINR values of all the receivers including the nodes in $W_{v}$ are all greater than $\beta$, then update $\left\langle S, t_{v}\right\rangle \in C T_{i}$ with $\left\langle S \cup\{v\}, t_{v}\right\rangle$.

(b) If any SINR value of the receivers including the nodes in $W_{v}$ is less than $\beta$, then the earliest transmission time for $v$ is $t_{v}+\Delta$. Increase $t_{v}$ by $\Delta$ and go to 1 .

Step 4.3. Update the broadcast schedule $S C H$ by $S C H=S C H \cup\left(U T_{i} \cup C T_{i}\right)$.

Based on the above four steps, we can generate broadcast schedules for BF-WSNs under the physical interference model.

\section{SIMULATION RESULTS}

This section evaluates the performances of the proposed algorithms by extensive simulations. In the simulations, we randomly deploy battery-free sensor nodes into a rectangular region with size $200 \mathrm{~m} \times 200 \mathrm{~m}$. The source sensor node is randomly chosen, since each sensor node has a chance to broadcast its data to other sensor nodes in the network. All sensor nodes have the same transmission radius of $25 \mathrm{~m}$. 
In the simulations, we use RF signal as the energy source. The time consumption for each sensor node to harvest $E_{f}$ energy, $T_{v}\left(E_{f}\right)$, is set within reasonable bounds [4, 31, 44]. According to References [31] and [4], the energy harvesting rates of battery-free sensor nodes harvesting energy from different RF sources range from $0.1 \mu \mathrm{W} / \mathrm{cm}^{2}$ to $100 \mu \mathrm{W} / \mathrm{cm}^{2}$. The energy consumption for a sensor node to receive and transmit a byte is $28.6 \mu \mathrm{J}$ and $59.2 \mu \mathrm{J}$, respectively, as in the Mica2dot platform [44]. Therefore, the time consumption $T_{v}\left(E_{f}\right)$ of $v$ to harvest enough energy for a transmission and a reception varies from 1 s to 1000 s, and we set $\Delta=1$ s. $\alpha$ is set as $\frac{1}{3}$.

According to the analysis in the previous sections, the broadcast latency of the broadcast schedules produced by the proposed algorithms are affected by two factors. The first one is the maximum time consumption for all battery-free sensor nodes to harvest enough energy for receiving and transmitting the broadcast data packet, $T_{\max }=\max \left\{T_{v}\left(E_{f}\right) \mid v \in V\right\}$. The second one is the depth of the tree generated by the Tran-Seq-Generator algorithm, $R$. The number of sensor nodes $n$, which indicates the scale of a BF-WSN may also affect the broadcast latency. In the following subsections, we will evaluate the impacts of $T_{\max }, R$, and $n$ on the broadcast latency, respectively.

According to the analysis in Section 1, all existing algorithms for solving the minimum-latency broadcast scheduling problem in traditional WSNs and duty-cycled WSNs are no longer suitable for BF-WSNs. Therefore, no off-the-shelf baseline methods can be compared with the proposed algorithms. To evaluate the performance of the proposed algorithms, we design the following method as the baseline.

The Baseline Method. First, all battery-free sensor nodes just harvest energy without any work during time $\left[t_{0}, t_{0}+T_{\max }\right]$, where $T_{\max }=\max \left\{T_{v}\left(E_{f}\right) \mid v \in V\right\}$. Therefore, all sensor nodes have accumulated enough energy to receive and relay the broadcast data packet at time $t_{0}+T_{\text {max }}$. Then, the broadcast schedule of a BF-WSN is the same as that of a traditional WSN. That is, the EBS algorithm [25] designed for broadcast scheduling in traditional WSNs is applied at time $t_{0}+T_{\max }$. This method is denoted as the baseline method in the following simulations.

\subsection{Impact of the Time $T_{\max }$}

We investigate the impact of time $T_{\max }$ on the broadcast latency of the broadcast schedules produced by the Baseline Method, the SBS algorithm, the WTSBS algorithm and the EWTSBS algorithm respectively. In this subsection, the depth of the tree generated by the Tran-Seq-Generator algorithm is fixed to $R=12$, and the network has $n=200$ battery-free sensor nodes in a region of $200 \mathrm{~m} \times 200 \mathrm{~m}$.

Five different BF-WSNs with the same size but different geographical topologies are tested. One hundred different groups of time consumption functions $\left\{T_{v}(e) \mid v \in V\right\}$ are applied for batteryfree sensor nodes in each geographical topology graph of the BF-WSN. Each data presented in this subsection is the average of the simulation results on 5 different BF-WSNs with 100 different groups of time consumption functions $\left\{T_{v}(e) \mid v \in V\right\}$.

In the first group of simulations, the maximum time consumption $T_{\max }$ for all battery-free sensor nodes to harvest enough energy for receiving and transmitting the broadcast data packet increases from 10 s to 100 s with increment 10 . The simulation results are shown in Figure 6. It shows that the broadcast latency increases with the growth of $T_{\max }$. Since $T_{\max }$ is relatively larger than $R$ in magnitude in our experimental setup, the differences among the performances of the proposed algorithms are not obvious in this figure. However, it is obviously presented that the broadcast latency of the broadcast schedule produced by the proposed algorithms are less than that of the Baseline Method. Furthermore, the broadcast latency of broadcast schedules produced by the WTSBS algorithm and the EWTSBS algorithm are less than that of the SBS algorithm, because the WTSBS algorithm and the EWTSBS algorithm can reduce the waiting time for sensor nodes in different 


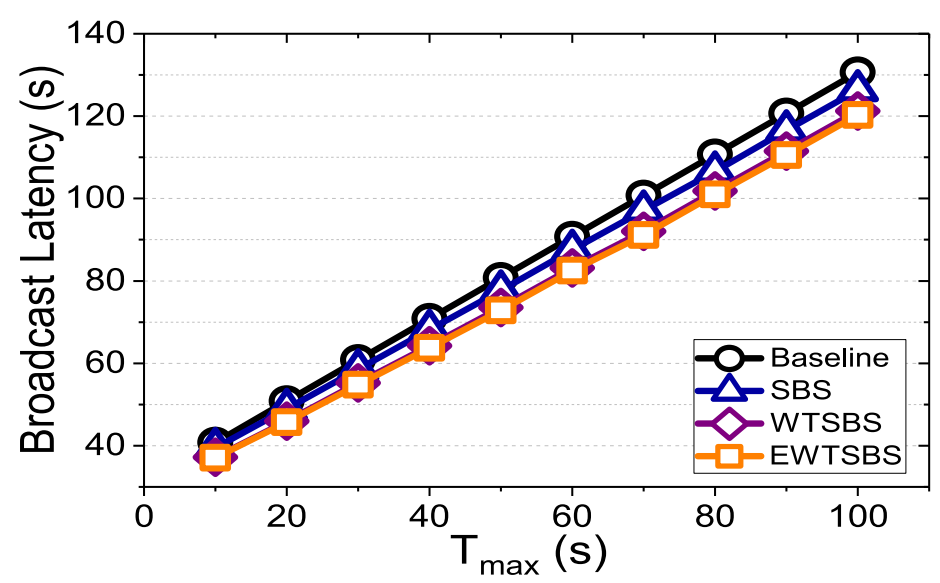

Fig. 6. The impact of time $T_{\max }$, where $T_{\max }$ ranges from $10 \mathrm{~s}$ to $100 \mathrm{~s}$.

Table 2. The Broadcast Latency of the Baseline Method

\begin{tabular}{cc}
\hline$T_{\max }(\mathbf{s})^{a}$ & The average broadcast latency $(\mathbf{s})$ \\
\hline 100 & 130.654 \\
200 & 230.214 \\
300 & 329.716 \\
400 & 429.232 \\
500 & 528.786 \\
600 & 628.286 \\
700 & 727.870 \\
800 & 827.170 \\
900 & 926.848 \\
1,000 & 1026.226 \\
\hline
\end{tabular}

$a_{\text {The maximum time consumption for battery-free sensor nodes to }}$ harvest $E_{f}$ amount of energy.

ways. Especially, the broadcast latency of broadcast schedule produced by the EWTSBS algorithm is the least.

In the second group of simulations, the performances of the proposed algorithms and the Baseline Method are evaluated with $T_{\max }$ increasing from 100 s to 1000 s with increment 100 . First, Table 2 shows the broadcast latency of the broadcast schedules produced by the Baseline Method, which is denoted by $T(B M)$. The broadcast latency of the broadcast schedules produced by the proposed algorithms are denoted by $T(S B S), T(W T)$, and $T(E W T)$, respectively.

For clarity, Figure 7 presents the reduced broadcast latency, i.e., $T(B M)-T(S B S), T(B M)-$ $T(W T)$, and $T(B M)-T(E W T)$. It is shown in Figure 7 that the reduced broadcast latency of every broadcast schedule produced by the proposed algorithms is positive. Therefore, the broadcast latency of the broadcast schedules produced by the proposed algorithms are less than that of the Baseline Method, which indicates the better performance of the proposed algorithms. Similarly to the first group of simulations, the broadcast latency of each broadcast schedule increases with the growth of $T_{\max }$. It can also be seen that the reduced broadcast latency $T(B M)-T(W T)$ and $T(B M)-T(E W T)$ are more than the reduced broadcast $T(B M)-T(S B S)$ as the WTSBS algorithm 


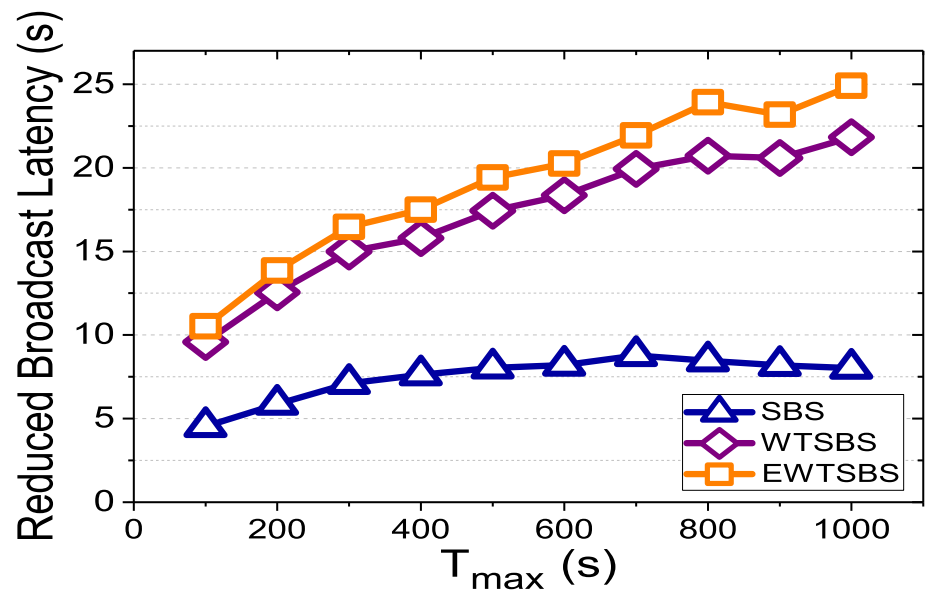

Fig. 7. The impact of time $T_{\max }$, where $T_{\max }$ ranges from $100 \mathrm{~s}$ to $1000 \mathrm{~s}$.

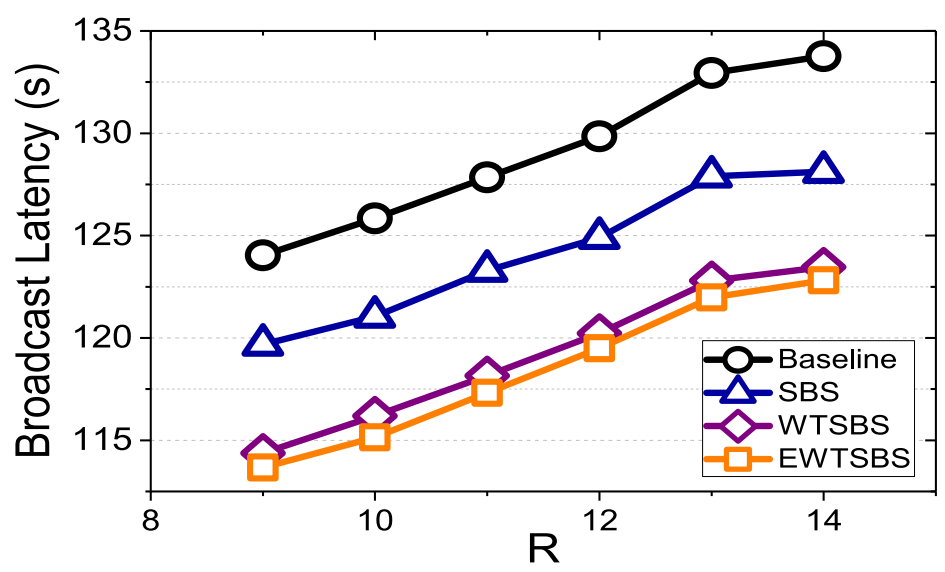

Fig. 8. The impact of the depth $R$ of tree $\mathcal{T}$.

and the EWTSBS algorithm can reduce the waiting time for sensor nodes in different ways. Especially, the reduced broadcast latency $T(B M)-T(E W T)$ is always the most.

\subsection{Impact of the Depth of Tree $\mathcal{T}$}

In this subsection, we evaluate the impact of $R$, the depth of the breadth-first searching tree $\mathcal{T}$ generated by the Tran-Seq-Generator algorithm, on the performances of the proposed algorithms. In the following simulations, the maximum time consumption of all battery-free sensor nodes to harvest enough energy for receiving and transmitting the broadcast data packet is fixed to $T_{\max }=100 \mathrm{~s}$. That is, all sensor nodes can harvest enough energy for receiving and transmitting the broadcast data packet after time $t_{0}+100$.

Ten different BF-WSNs with the same size $(n=200)$ but different geographical topologies are tested. One hundred different groups of time consumption functions $\left\{T_{v}(e) \mid v \in V\right\}$ are applied, where $T_{v}\left(E_{f}\right) \leq T_{\max }=100, \forall v \in V$. The simulation results are presented in Figure 8. Each datum presented in this subsection is the average of simulation results on 10 different BF-WSNs with 100 different groups of time consumption functions $\left\{T_{v}(e) \mid v \in V\right\}$. 


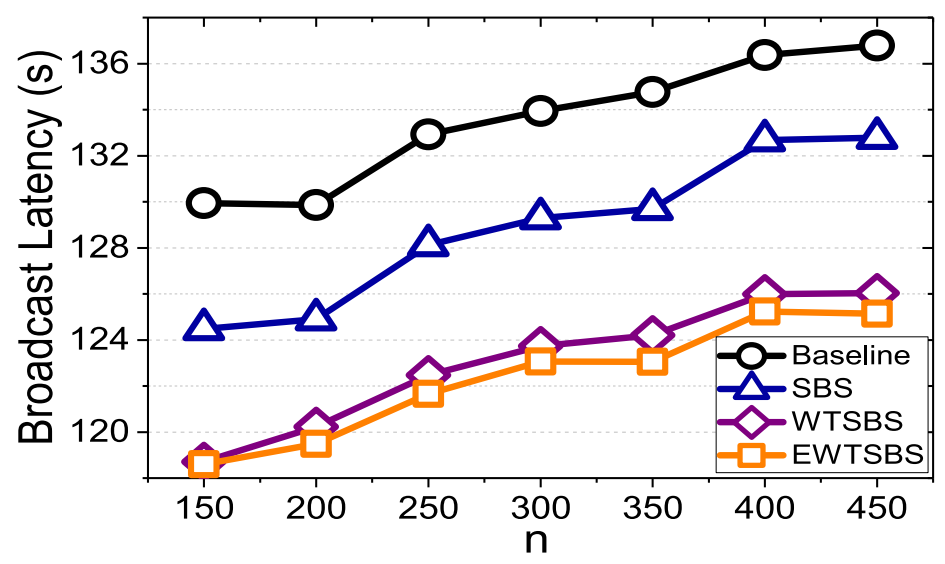

Fig. 9. The impact of network size.

According to Figure 8, the broadcast latency of broadcast schedules produced by the Baseline Method, the SBS algorithm, the WTSBS algorithm and the EWTSBS algorithm increase with the growth of $R$. The larger $R$ implies that there are more hops between the source sensor node and the farthest sensor node in the BF-WSN, which increases the latency of the communication between them. Therefore, a larger $R$ increases broadcast latency. It is also shown that the broadcast latency of the broadcast schedule produced by the SBS algorithm is larger than that of the WTSBS algorithm and the EWTSBS algorithm. The EWTSBS algorithm has the best performance among the three proposed algorithms, and all these three algorithms outperform the Baseline Method.

\subsection{Impact of Network Size}

Finally, we study the performance of the algorithms while network size varies. The depth of the breadth-first searching tree generated by the Tran-Seq-Generator algorithm is fixed to $R=12$ and the maximum time consumption of all battery-free sensor nodes to harvest enough energy for receiving and transmitting the broadcast data packet is fixed to $T_{\max }=100 \mathrm{~s}$. The number of sensor nodes in the BF-WSNs varies from 150 to 450 with increment 50.

For each network size, 10 different BF-WSNs with the same size but different geographical topologies are tested. 100 different groups of time consumption functions $\left\{T_{v}(e) \mid v \in V\right\}$ are applied, where $T_{v}\left(E_{f}\right) \leq T_{\max }=100, \forall v \in V$. Figure 9 shows the simulation results, where each data presented in this subsection is the average of simulation results on 10 different BF-WSNs with 100 different groups of time consumption functions $\left\{T_{v}(e) \mid v \in V\right\}$.

As shown in Figure 9, the broadcast latency of all broadcast schedules produced by all algorithms increase with the growth of the network size. More sensor nodes in the fixed $200 \mathrm{~m} \times 200 \mathrm{~m}$ region will cause more collisions. Therefore, to avoid collisions, the broadcast latency of the broadcast schedule will increase. No matter how many sensor nodes are there in a BF-WSN, the broadcast latency of of broadcast schedules produced by the proposed algorithms are always better than that of the Baseline Method. The EWTSBS algorithm has the best performance among three proposed algorithms.

\section{RELATED WORKS}

Broadcast is an essential operation in WSNs, which has attracted substantial research interest. The problem of minimum energy broadcast is studied in Reference [6], where the NP-completeness of 
the problem is proved. A transparent link correlation layer, called CorLayer, is proposed in Reference [48] for energy efficient broadcast. The authors in Reference [49] focus on devising backbone forwarders selecting metrics for broadcast in WSNs. Network coding is considered in broadcast forwarder selection to further reduce unnecessary transmissions. Particularly, the minimumlatency broadcast scheduling problem in WSNs has been studied extensively for a long time [10, 17, 18, 21, 22, 24-26, 29, 32, 41, 43, 46, 47]. It has been proved to be NP-hard in Reference [18]. Numerous approximation algorithms have been proposed to design collision-free broadcast schedules for WSNs with minimum broadcast latency, which can be divided into two categories.

The first category of algorithms are proposed to design minimum-latency broadcast schedules for traditional WSNs, where all sensor nodes in a network are powered by batteries and are always active in their lifetime. An approximation algorithm with approximation ratio of 400 is proposed in Reference [18]. Three approximation algorithms with latency at most $24 R-23,16 R-15$ and $R+$ $O(\log R)$ are proposed in Reference [25]. An approximation algorithm with approximation ratio of 12 for the one-to-all broadcast problem and two algorithms with approximation ratios of 20 and 34 for the all-to-all broadcast problem are proposed in Reference [17]. Different from the above works, the work in Reference [10] considers the case of interference range being strictly larger than the transmission radius, and proposes an approximation algorithm with approximation ratio of $\mathrm{O}\left(\alpha^{2}\right)$, where $\alpha>1$ is the ratio of the interference range to the transmission radius. Besides interference range, the work in Reference [32] further considers the carrier sensing radius and proposes an approximation algorithm for collision-free broadcast schedule. The broadcast scheduling problem under two models, 2-Disk model and SINR-model, are investigated in Reference [24]. A constant approximation algorithm is proposed for the 2-Disk model and it is extended to the SINR model. The broadcast scheduling problems in both 2D and 3D WSNs are studied in Reference [43], and several approximation algorithms for the problems are proposed.

The second category of algorithms are proposed to design minimum-latency broadcast schedules for duty-cycled WSNs, where each sensor node has fixed time slots during its lifetime for receiving a data packet and can be woken up at any time to transmit a data packet. The NPhardness of the minimum-latency broadcast scheduling problem in duty-cycled WSNs is proved in Reference [21]. The ELAC algorithm with an approximation ratio of $24 T+1$ is proposed, where $T$ denotes the number of time slots in a working period. The approximation ratio has been improved to $17 T$ by the OTAB algorithm in Reference [26]. The LABS scheme that further reduces the broadcast latency is proposed in Reference [29]. The improvement is achieved by optimizing the transmissions from a sensor node to each of its neighbors. The broadcast scheduling over asynchronous duty-cycling in WSNs is considered in Reference [46]. The problem is first transformed into a shortest-path problem in a time-coverage graph, and then two similar distributed algorithms are proposed. Another Asynchronous Duty-Cycle Broadcasting protocol without relying on any broadcast tree is proposed in Reference [41]. In Reference [22], the authors prove that the problem of minimizing broadcast redundancy in duty-cycled WANETs is NP-hard and propose a centralized algorithm with an approximation ratio of $3(\ln \Delta+1)$, where $\Delta$ denotes the maximum node degree in a network. In Reference [47], it is proved that the problem of balancing the number of transmissions and latency in a broadcast schedule is NP-hard. A centralized dynamic programming algorithm is proposed to solve this problem.

Unfortunately, all these existing algorithms for solving the minimum-latency broadcast scheduling problem are no longer suitable for BF-WSNs due to the special characteristics of BF-WSNs as discussed in Section 1. The battery-free sensor nodes in a BF-WSN harvest energy from ambient environment instead of powered by batteries so that they cannot always stay in active state and are often out of service. Therefore, in the transmission and reception time designed by the first category of algorithms for traditional WSNs and the second category of algorithms for duty-cycled 
WSNs, the battery-free sensor nodes may not have enough energy to transmit or receive a data packet.

\section{CONCLUSION}

In this article, we investigate the minimum-latency broadcast scheduling problem in BF-WSNs. Firstly, we formally define the MLBS-BF problem and prove its NP-hardness. Then, three approximation algorithms, the SBS algorithm, the WTSBS algorithm and the EWTSBS algorithm, are proposed. The correctness of the proposed algorithms are proved and the broadcast latency bounds of the broadcast schedules generated by the proposed algorithms are analyzed. Furthermore, the approximation ratio of each proposed algorithm is analyzed. Besides, the broadcast scheduling for BF-WSNs under physical interference model is discussed. Finally, extensive simulations are carried out to evaluate the performances of the proposed algorithms. The simulation results show that the proposed algorithms are efficient and effective.

\section{REFERENCES}

[1] Mark G. Ball, Blerim Qela, and Slawomir Wesolkowski. 2016. A review of the use of computational intelligence in the design of military surveillance networks. In Recent Advances in Computational Intelligence in Defense and Security. Springer, 663-693.

[2] Barbara Barbagli, Luca Bencini, Iacopo Magrini, Gianfranco Manes, and Antonio Manes. 2011. A real-time traffic monitoring based on wireless sensor network technologies. In Proceedings of the 2011 th International Wireless Communications and Mobile Computing Conference (IWCMC'11). IEEE, 820-825.

[3] Carlo Bergonzini, Davide Brunelli, and Luca Benini. 2009. Algorithms for harvested energy prediction in batteryless wireless sensor networks. In Proceedings of the 3rd International Workshop on Advances in Sensors and Interfaces 2009 (IWASI'09). IEEE, 144-149.

[4] Kenneth Brown, Jason Reese, and Jian Zhen. 2012. RF energy harvesting. University of Cincinnati. B.S. Thesis.

[5] Michael Buettner, Ben Greenstein, and David Wetherall. 2011. Dewdrop: An energy-aware runtime for computational RFID. In Proceedings of the USENIX Symposium on Networked Systems Design and Implementation (NSDI'11). 197-210.

[6] Mario Čagalj, Jean-Pierre Hubaux, and Christian Enz. 2002. Minimum-energy broadcast in all-wireless networks: NPcompleteness and distribution issues. In Proceedings of the 8th Annual International Conference on Mobile Computing and Networking. ACM, 172-182.

[7] Zhipeng Cai and Zaobo He. 2019. Trading private range counting over big IoT data. In Proceedings of the 39th IEEE International Conference on Distributed Computing Systems (ICDCS'19). IEEE.

[8] Alessandro Cammarano, Chiara Petrioli, and Dora Spenza. 2012. Pro-energy: A novel energy prediction model for solar and wind energy-harvesting wireless sensor networks. In Proceedings of the 2012 IEEE 9th International Conference on Mobile Ad-Hoc and Sensor Systems (MASS'12). IEEE, 75-83.

[9] Kunyi Chen, Hong Gao, Zhipeng Cai, Quan Chen, and Jianzhong Li. 2019. Distributed energy-adaptive aggregation scheduling with coverage guarantee for battery-free wireless sensor networks. In Proceedings of the IEEE Conference on Computer Communications (INFOCOM'19). IEEE, 1018-1026.

[10] Zhenming Chen, Chunming Qiao, Jinhui Xu, and Taekkyeun Lee. 2007. A constant approximation algorithm for interference aware broadcast in wireless networks. In Proceedings of the 26th IEEE International Conference on Computer Communications (INFOCOM'07). IEEE, 740-748.

[11] Siyao Cheng, Zhipeng Cai, and Jianzhong Li. 2015. Curve query processing in wireless sensor networks. IEEE Trans. Vehic. Technol. 64, 11 (2015), 5198-5209.

[12] Siyao Cheng, Zhipeng Cai, Jianzhong Li, and Xiaolin Fang. 2015. Drawing dominant dataset from big sensory data in wireless sensor networks. In Proceedings of the 2015 IEEE Conference on Computer Communications (INFOCOM'15). IEEE, 531-539.

[13] Siyao Cheng, Zhipeng Cai, Jianzhong Li, and Hong Gao. 2017. Extracting kernel dataset from big sensory data in wireless sensor networks. IEEE Trans. Knowl. Data Eng. 29, 4 (2017), 813-827.

[14] Gustavo Medeiros de Araújo, Alex R. Pinto, Jörg Kaiser, and Leandro Buss Becker. 2014. Genetic machine learning approach for link quality prediction in mobile wireless sensor networks. In Cooperative Robots and Sensor Networks. Springer, 1-18.

[15] Giacomo De Meulenaer, François Gosset, François-Xavier Standaert, and Olivier Pereira. 2008. On the energy cost of communication and cryptography in wireless sensor networks. In Proceedings of the IEEE International Conference on Wireless and Mobile Computing, Networking, and Communications 2008 (WIMOB'08). IEEE, 580-585. 
[16] Aoife M. Foley, Paul G. Leahy, Antonino Marvuglia, and Eamon J. McKeogh. 2012. Current methods and advances in forecasting of wind power generation. Renew. Energy 37, 1 (2012), 1-8.

[17] Rajiv Gandhi, Yoo-Ah Kim, Seungjoon Lee, Jiho Ryu, and Peng-Jun Wan. 2012. Approximation algorithms for data broadcast in wireless networks. IEEE Trans. Mobile Comput. 11, 7 (2012), 1237-1248.

[18] Rajiv Gandhi, Arunesh Mishra, and Srinivasan Parthasarathy. 2008. Minimizing broadcast latency and redundancy in ad hoc networks. IEEE/ACM Trans. Netw. 16, 4 (2008), 840-851.

[19] Tian He, Sudha Krishnamurthy, Liqian Luo, Ting Yan, Lin Gu, Radu Stoleru, Gang Zhou, Qing Cao, Pascal Vicaire, John A. Stankovic, et al. 2006. VigilNet: An integrated sensor network system for energy-efficient surveillance. ACM Trans. Sens. Netw. 2, 1 (2006), 1-38.

[20] Zaobo He, Zhipeng Cai, Siyao Cheng, and Xiaoming Wang. 2015. Approximate aggregation for tracking quantiles and range countings in wireless sensor networks. Theor. Comput. Sci. 607 (2015), 381-390.

[21] Jue Hong, Jiannong Cao, Wenzhong Li, Sanglu Lu, and Daoxu Chen. 2009. Sleeping schedule-aware minimum latency broadcast in wireless ad hoc networks. In Proceedings of the IEEE International Conference on Communications 2009 (ICC'09). IEEE, 1-5.

[22] Jue Hong, Jiannong Cao, Wenzhong Li, Sanglu Lu, and Daoxu Chen. 2010. Minimum-transmission broadcast in uncoordinated duty-cycled wireless ad hoc networks. IEEE Trans. Vehic. Technol. 59, 1 (2010), 307-318.

[23] Wen Hu, Nirupama Bulusu, Chun Tung Chou, Sanjay Jha, Andrew Taylor, and Van Nghia Tran. 2009. Design and evaluation of a hybrid sensor network for cane toad monitoring. ACM Trans. Sens. Netw. 5, 1 (2009), 4.

[24] Scott C.-H. Huang, Peng-Jun Wan, Jing Deng, and Yunghsiang S. Han. 2008. Broadcast scheduling in interference environment. IEEE Trans. Mobile Comput. 7, 11 (2008), 1338-1348.

[25] S. C.-H. Huang, P.-J. Wan, Xiaohua Jia, Hongwei Du, and Weiping Shang. 2007. Minimum-latency broadcast scheduling in wireless ad hoc networks. In Proceedings of the 26th IEEE International Conference on Computer Communications (INFOCOM'07). IEEE, 733-739.

[26] Xianlong Jiao, Wei Lou, Junchao Ma, Jiannong Cao, Xiaodong Wang, and Xingming Zhou. 2012. Minimum latency broadcast scheduling in duty-cycled multihop wireless networks. IEEE Trans. Parallel Distrib. Syst. 23, 1 (2012), 110117.

[27] Aman Kansal, Jason Hsu, Sadaf Zahedi, and Mani B. Srivastava. 2007. Power management in energy harvesting sensor networks. ACM Trans. Embed. Comput. Syst. 6, 4 (2007), 32.

[28] Sukun Kim, Shamim Pakzad, David Culler, James Demmel, Gregory Fenves, Steven Glaser, and Martin Turon. 2007. Health monitoring of civil infrastructures using wireless sensor networks. In Proceedings of the 6th International Conference on Information Processing in Sensor Networks. ACM, 254-263.

[29] Duc Tai Le, Thang Le Duc, Vyacheslav V. Zalyubovskiy, Dongsoo S. Kim, and Hyunseung Choo. 2014. LABS: Latency aware broadcast scheduling in uncoordinated duty-cycled wireless sensor networks. F. Parallel Distrib. Comput. 74, 11 (2014), 3141-3152.

[30] Mo Li, Yunhao Liu, and Lei Chen. 2008. Nonthreshold-based event detection for 3D environment monitoring in sensor networks. IEEE Trans. Knowl. Data Eng. 20, 12 (2008), 1699-1711.

[31] Xiao Lu, Ping Wang, Dusit Niyato, Dong In Kim, and Zhu Han. 2015. Wireless networks with RF energy harvesting: A contemporary survey. IEEE Commun. Surv. Tutor. 17, 2 (2015), 757-789.

[32] Reza Mahjourian, Feng Chen, Ravi Tiwari, My Thai, Hongqiang Zhai, and Yuguang Fang. 2008. An approximation algorithm for conflict-aware broadcast scheduling in wireless ad hoc networks. In Proceedings of the 9th ACM International Symposium on Mobile ad Hoc Networking and Computing. ACM, 331-340.

[33] Kapileswar Nellore and Gerhard P. Hancke. 2016. A survey on urban traffic management system using wireless sensor networks. Sensors 16, 2 (2016), 157.

[34] Prusayon Nintanavongsa, Ufuk Muncuk, David Richard Lewis, and Kaushik Roy Chowdhury. 2012. Design optimization and implementation for RF energy harvesting circuits. IEEE f. Emerg. Select. Top. Circ. Syst. 2, 1 (2012), 24-33.

[35] Hiroshi Nishimoto, Yoshihiro Kawahara, and Tohru Asami. 2010. Prototype implementation of ambient RF energy harvesting wireless sensor networks. In Proceedings of the 2010 IEEE Conference on Sensors. IEEE, 1282-1287.

[36] Joaquin Recas Piorno, Carlo Bergonzini, David Atienza, and Tajana Simunic Rosing. 2009. Prediction and management in energy harvested wireless sensor nodes. In Proceedings of the 1st International Conference on Wireless Communication, Vehicular Technology, Information Theory and Aerospace \& Electronic Systems Technology 2009 (Wireless VITAE'09). IEEE, 6-10.

[37] Tuo Shi, Zhipeng Cai, Jianzhong Li, and Hong Gao. 2019. The energy-data dual coverage in battery-free sensor networks. In Proceedings of the 39th IEEE International Conference on Distributed Computing Systems (ICDCS'19). IEEE.

[38] Tuo Shi, Siyao Cheng, Jianzhong Li, and Zhipeng Cai. 2017. Constructing connected dominating sets in battery-free networks. In Proceedings of the IEEE Conference on Computer Communications (INFOCOM'17). IEEE, 1-9.

[39] Tuo Shi, Siyao Cheng, Jianzhong Li, Hong Gao, and Zhipeng Cai. 2019. Dominating sets construction in RF-based battery-free sensor networks with full coverage guarantee. ACM Trans. Sens. Netw. 
[40] Tuo Shi, Jianzhong Li, Hong Gao, and Zhipeng Cai. 2018. Coverage in battery-free wireless sensor networks. In Proceedings of the IEEE Conference on Computer Communications (INFOCOM'18). IEEE, 108-116.

[41] Yanjun Sun, Omer Gurewitz, Shu Du, Lei Tang, and David B. Johnson. 2009. ADB: An efficient multihop broadcast protocol based on asynchronous duty-cycling in wireless sensor networks. In Proceedings of the 7th ACM Conference on Embedded Networked Sensor Systems. ACM, 43-56.

[42] Xiang Tian, Jiguo Yu, Liran Ma, Guangshun Li, and Xiuzhen Cheng. 2016. Distributed deterministic broadcasting algorithms under the sinr model. In Proceedings of the 35th Annual IEEE International Conference on Computer Communications (INFOCOM'16). IEEE, 1-9.

[43] Ravi Tiwari, Thang N. Dinh, and My T. Thai. 2013. On centralized and localized approximation algorithms for interference-aware broadcast scheduling. IEEE Trans. Mobile Comput. 12, 2 (2013), 233-247.

[44] Arvinderpal S. Wander, Nils Gura, Hans Eberle, Vipul Gupta, and Sheueling Chang Shantz. 2005. Energy analysis of public-key cryptography for wireless sensor networks. In Proceedings of the 3rd IEEE International Conference on Pervasive Computing and Communications 2005 (PerCom'05). IEEE, 324-328.

[45] Demin Wang, Bin Xie, and Dharma P. Agrawal. 2008. Coverage and lifetime optimization of wireless sensor networks with gaussian distribution. IEEE Trans. Mobile Comput. 7, 12 (2008), 1444-1458.

[46] Feng Wang and Jiangchuan Liu. 2009. Duty-cycle-aware broadcast in wireless sensor networks. In Proceedings of the Annual IEEE International Conference on Computer Communications (INFOCOM'09). IEEE, 468-476.

[47] Feng Wang and Jiangchuan Liu. 2012. On reliable broadcast in low duty-cycle wireless sensor networks. IEEE Trans. Mobile Comput. 11, 5 (2012), 767-779.

[48] Shuai Wang, Song Min Kim, Yunhuai Liu, Guang Tan, and Tian He. 2013. Corlayer: A transparent link correlation layer for energy efficient broadcast. In Proceedings of the 19th Annual International Conference on Mobile Computing \& Networking. ACM, 51-62.

[49] Shuai Wang, Guang Tan, Yunhuai Liu, Hongbo Jiang, and Tian He. 2014. Coding opportunity aware backbone metrics for broadcast in wireless networks. IEEE Trans. Parallel Distrib. Syst. 25, 8 (2014), 1999-2009.

[50] Ning Xu, Sumit Rangwala, Krishna Kant Chintalapudi, Deepak Ganesan, Alan Broad, Ramesh Govindan, and Deborah Estrin. 2004. A wireless sensor network for structural monitoring. In Proceedings of the 2nd International Conference on Embedded Networked Sensor Systems. ACM, 13-24.

[51] Jiguo Yu, Baogui Huang, Xiuzhen Cheng, and Mohammed Atiquzzaman. 2017. Shortest link scheduling algorithms in wireless networks under the SINR model. IEEE Trans. Vehic. Technol. 66, 3 (2017), 2643-2657.

[52] Jiguo Yu, Shengli Wan, Xiuzhen Cheng, and Dongxiao Yu. 2017. Coverage contribution area based $k$-coverage for wireless sensor networks. IEEE Trans. Vehic. Technol. 66, 9 (2017), 8510-8523.

Received July 2018; revised June 2019; accepted August 2019 